FINAL REPORT

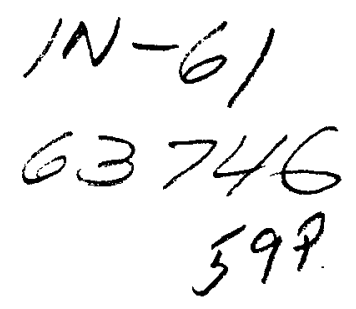

FOR GRANT NSG-1555

\title{
INVESTIGATION OF SCENE IDENTIFICATION ALGORITHMS \\ FOR RADIATION BUDGET MEASUREMENTS
}

by

F. J. DIEKMANN

GEORGE WASHINGTON UNIVERSITY

HAMPTON, VIRGINIA

TECHNICAL MONITOR:

G. L. SMITH

ATMOSPHERIC SCIENCES DIVISION

NASA, LANGLEY RESEARCH CENTER

HAMPTON, VIRGINIA

DECEMBER, 1986 


\title{
INVESTIGATION OF SCENE IDENTIFICATION ALGORITHMS \\ FOR RADIATION BUDGET MEASUREMENTS
}

\author{
by \\ F. J. Diekmann \\ George Washington University, Hampton, Virginia \\ ARSTRACT
}

The computation of Earth radiation budget from satellite measurements requires the identification of the scene in order to select spectral factors and bidirectional models. In order to evaluate the effects of scene identification errors on the resulting radiant exitances, scene identification probability matrices, which quote the probability that a scene which is in truth type $j$ is identified as type $i$, are needed. This paper presents empirical evaluations of these matrices by intercomparing preliminary results from the ERBe scene identification alyorithm with resuits using Avinn data.

A scene identification procedure is developed fọ AVHRR SW and LW data by using two radiative transfer models. These AVHRR GAC pixels are then attached to corresponding ERBE pixels and the results are sorted into scene identification probability matrices. These scene intercomparisons show that there generaliy is a higher tendency for underestimation of cloudiness over ocean at high cloud amounts, e.g. mostly cloudy instead of overcast partly cloudy instead of mostly cloudy, for the ERBE relative to the AVHRR results. Reasons for this are explained.

Preliminary estimates of the errors of exitances due to scene misidentification demonstrates the high dependency on the probability matrices. While the longwave error can generally be neglected the shortwave deviations have reached maximum values of more than $12 \%$ of the respective exitances. Numerical results in this paper must be upgraded with the final ERBE data when they become available. 


\title{
Investigation of Scene Identification Algorithms \\ for Radiation Rudget Measurements
}

by

\author{
F. J. Diekmann \\ George Washington University \\ Hampton, Virginia \\ and \\ G. L. Smith \\ Atmospheric Sciences Division \\ NASA, Langley Research Center \\ Hampton, Virginia
}

\section{INTRODUCTION}

The Earth Radiation Budget Experiment (ERBE) is a major effort in the understanding of the Earth's climate and its variations (Barkstrom and Smith, 1986). With ERBE, as in any satellite experiment for study of Earth energy budget at medium spatial scales, a scanning radiometer measures the radiance in one direction due to the upwelling reflected solar (shortwave) radiation or the Earth emitted (1ongwave) radiation. In order to compute the radiant exitance in either of these ranges, it is necessary to account for the isotropy of the radiation, which is described hy a limb-darkening function for Earth emitted radiation and a bidirectional function for reflected solar radiation. This approach was used for the Nimbus ? and Nimbus 3 Medium Resolution Infrared Radiometer (Raschke and Bandeen, 1970; Raschke et al., 1973), the Nimbus 6 and 7 Earth Radiation Budget (W. L. Smith et al., 1978; Jacohowitz et al., 1984), and currently the ERBE (G. L. Smith et al., 1986). These functions depend upon the directions of the incoming and exiting rays, and also upon the scene, by which we mean the Earth's surface and the intervening atmosphere. The scenes are classified into a small number of scene types. The computation of radiant exitance 
from the radiance measurement thus requires first the identification of the scene as one of these types, and the selection of the corresponding bidirectional and limb-darkening function. Due to the importance of these functions, their experimental determination as was done by Taylor and Stowe (1984) was one of the major objectives of the ERB experiment on the Nimbus 6 and 7 spacecraft. Furthermore, much computation has been done to define these functions theoretically, e.g. as by Stuhlmann et al. (1985).

There is a second reason for the need for scene identification in the determination of Earth radiation budget. A scanning radiometer for Earth radiation budget ideally measures the broadband radiance in the shortwave and in the longwave ranges. However, the optics of any real radiometer will not have the ideal spectral response across the spectrum to as sharp cutoff, but will have a more complex spectral response. For example, the spectral responses of the three channels of the ERBE scanning radiometer are rather complicated functions of wavelength, as shown in Figure 1 . The spectral responses of the shortwave and longwave channels of the ERB scanning radiometers aboard the Nimbus 6 and 7 spacecraft are essentially the same as these of the ERBE. For the ERBE data analysis, the spectral responses of the channels are accounted for by identifying the scene, which in turn provides an approximation of the incoming spectrum. From this information, the broadband radiances can be accurately computed (Avis et al., 1984).

Because of the importance of scene identification in the data analysis process, it is necessary to understand the algorithm by which the scene is identified. This paper presents a study of the ERBE scene identification algorithm and helps to define its strengths and weaknesses. Similar considerations may be expected for any radiation budget measurements by satellite. The approach in this study is to use radiative transfer theory to 
establish a quantitative understanding of the various parameters of the problem. These theoretical results are compared with satellite data as a test of this understanding with real data.

The performance of a scene identification algorithm is measured objectively by the probability that a scene which is type $i$ is computed to be type $j$. These probabilities for all combinations of $i$ and $j$ form a matrix. This scene identification matrix is shown by Smith et al. (1984) to be important in the calculation of error propagation and generation in the analysis of Earth radiation budget data. The present paper provides scene identification probability matrices on the basis of the comparison of ERBE derived scenes compared with the scenes as derived from AVHRR data. Because the scene identifications from the AVHRR are themselves subject to error, these probabilities are not with respect to absolute truth, but with respect to agreement of the two algorithms. Thus, as with any experimentally based quantities, these matrices are approximations. However, they are the first available. As such, they permit the computation for the first time of the errors in shortwave and longwave radiant exitances which are due to scene identification errors as measured by these scene identification probability matrices. Preliminary results for these radiant exitance errors are presented.

Because they are based on preliminary ERRE data tapes which are in the process of being revised, all results in this paper are preliminary. Indeed, a major reason for the work is to provide information concerning the performance of the ERBE scene identification algorithm so as to permit improvements to the algorithm prior to finalizing it for production of valid ERBE data. For this reason, the numerical results in this paper must be upgraded with the final ERBE data. 


\section{APPROACH}

We begin with a review of the ERBE scene identification algorithm and its role in data analysis. The basic requirements for a simulation study of the algcrithm are thus defined, and the approach is designed. In order to study the application of the algorithm with real data, AVHRR data are used as "truth" against which the performance of the ERRE i.s compared. A. nescription of ERRE scene identification algorithm

The data flow for analysis of scanning radiometer data is set up as shown in Figure 2. Using "global" spectral factors, approximate broadband shortwave and longwave radiances $L$ at the spacecraft are computed. The scene is then identified and the appropriate spectral factors are determined for the final calculation of the radiances. Also, a bidirectional model $R$ based on this scene type is used to account for anisotropy of the radiation in the estimate of the radiant exitance $M$ for the pixei, i.e. the fieid of view of a single measurement, by use of equation .(1):

$$
M=\pi L / R
$$

The scene identification procedure for ERBE first classifies the underlying surface by its geographic type (geotype): land, ocean, snow, desert or land/ocean mix (coastal regions). The cloud cover is defined in terms of four classes: clear (less than 5\% cloud cover), partly cloudy (between $5 \%$ and $50 \%$ cloud), mostly cloudy (between $50 \%$ and $95 \%$ cloud) and overcast (more than $95 \%$ cloudy).

The cloud class for a given measurement pair (shortwave and longwave radiances) is selected according to the measurement pair in a manner similar to a threshold method, but modified to account for the variance associated with each class, as illustrated in Figure 3. There is a distribution of measurement pairs within each class, as represented by the light lines. The 
shortwave-longwave plane is partitioned into regions according to which is the most probable scene identification for the measurement pair. The boundaries of these regions are indicated by the heavy solid lines in Figure 3 . Thus, if a measurement pair falls within region I, given that the surface is ocean, it is identified as "clear ocean". For nighttime scene identification, the cloud class assignment is made according to the longwave radiance only, in a manner analogous to the daytime case.

B. Simulation approach

It is seen that the ERBE scene identification algorithm is a bispectral technique using SW and LW measurements. In order to simulate the operation of the scene identification algorithm for a given scene, it is necessary to compute the SW and $L W$ measurements for the scene. The measurement for a radiometer channel is given by

$$
m^{c}=\int_{0}^{\infty} S_{\lambda}^{c} L_{\lambda} d \lambda
$$

where $S_{\lambda}{ }^{C}$ is the spectral response of channel $c$. In this paper, radiative transfer theory is used to compute spectral radiances for a variety of scenes, and the resulting measurements will be computed for the ERRE scanning radiometer channels and for the AVHRR channels using equation (2).

Shortwave and longwave spectral radiances are computed for a number of model atmospheres and surfaces. In order to avoid excessive computational requirements, a two stream approximation is used to compute the shortwave spectral radiant exitances. The spectral radiances are then calculated by use of spectral bidirectional models $R_{\lambda}$ which were computed by Stuhlmann et a . (1985) and stored on tape. These SW and LW spectral radiances constitute the Level I data. The measurements were then computed for a given radiometer channel by use of these spectral radiances and its spectral response in equation (2), and make up the Level II data. Finally, the means, 
variances and correlation coefficients are computed for this set of SW and LW measurements. These statistics are the Level III data set, which is required for the scene identification algorithm. Because the model atmospheres and surfaces for the radiance calculations were not statistically distributed, this procedure also does not yield a statistically valid distribution of scenes and their variances, which would be desireable. However, it does provide reasonable numbers for the shortwave and longwave means, their variances and correlations, which are needed for the scene identification algorithm. The reasonableness of the approach is demonstrated by comparison of the Level III results with ERBE and AVHRR measurements. The AVHRR measurements are from the $V 5$ tape, which is an ERBE produced magnetic tape for validation. This tape contains concurrent measurements from the ERBE and AVHRR instruments on board the NOAA 9 spacecraft. In order to provide a truth set for comparison with the ERBE scene identifications, the maximum likelihood method is adapted to the AVHRR data. In addition, the method of Eyre et al. (1984) is used with the AVHRR data for cloud discrimination to provide assurance that the AVHRR scene identifications are reliable. It is then determined which AVHRR pixels fit within a given ERBE pixel, and the probabilities are computed for the scene identification of the ERBE. Finally, the probability matrices for the scene identification are used for the evaluation of the errors which are incurred in data inversion (Smith et al., 1984). The approach is shown schematically in figure 4 . 
III. COMPUTATION OF THEORETICAL FILTEREN RADIANCES

In the computation of $L_{\lambda}$, it is assumed that radiation in the shortwave spectral range is reflected solar radiation and that radiation in the longwave is Earth-atmosphere emitted radiation only.

III A. Radiative Transfer Computations

For LW radiation, scattering is neglected and the surface is assumed to be perfectly absorhing. Thus, the spectral radiance along a ray at a given zenith angle is given by integrating the absorption and emission along the ray. Computations are made for each of nine zenith angles: $0^{\circ}, 10^{\circ}, \ldots, 80^{\circ}$. Because solar radiation does not contribute in this spectral range, there is no dependence on $\theta_{0}$ and $\phi$. Spectral radiances are computed as described by Schmetz and Raschke (1981). Brief1y, the LW model computes spectral radiances in each of 51 spectral intervals, ranging from 4 to $400 \mu \mathrm{m}$. Absorption and emission by $\mathrm{H}_{2} \mathrm{O}, \mathrm{O}_{3}, \mathrm{H}_{2} \mathrm{O}$ polymers and "uniformly mixed gases" (i.e. $\mathrm{CO}_{2}, \mathrm{CH}_{4}, \mathrm{~N}_{2} \mathrm{O}$ ) and in clouds, water droplets are taken into account. Longwave radiant exitance is next computed from these spectral radiances by integrating over the zenith angle and the spectral ranges.

For the computation of SW radiances, in the range 0.20 to $3.7 \mu \mathrm{m}$, multiple scattering must be considered. In order to reduce the computational requirements, spectral radiant exitances are computed hy use of the twostream approximation and the spectral bidirectional models of Stuhlmann et al. (1985) is applied to all SW wavelengths to get spectral radiances. The computation of SW spectral fluxes includes Rayleigh and aerosol scattering and absorption by water vapor, aerosols, ozone, the "uniformly mixed gases" ( $\mathrm{i} . \mathrm{e} . \mathrm{CO}_{2}, \mathrm{CH}_{4}, \mathrm{~N}_{2} \mathrm{O}$ ), and in clouds, water droplets. Transmission functions for the atmospheric gases are taken from Kerschgens et al. (1976), and aerosol radiative properties are those recommended by IAMAP (MCClatchey 
et a1., 1977). The spectral reflectivity of the lower boundary was treated as by Kerschgens et al. (1976).

Cloud was considered to be a one kilometer thick homogeneous layer within the atmosphere, with variable cloud top heights from one to $15 \mathrm{~km}$. The cloud droplet size distribution is the Sc-2 from Schmetz et al. (1981) as measured in stratocumulus clouds, with an effective droplet radius of $7.9 \mu \mathrm{m}$. The radiative properties, including single scattering albedo and asymmetry factor are the same as those computed by Stuhlmann et al. (1985), using Mie theory. The optical depth at $\lambda=.55 \mu \mathrm{m}$ was determined from the droplet size distribution, and the optical depth at other wavelengths was then computed, based on Schmetz et al (1981). Varying the cloud optical depth between 1 and 100 results in a range of column liquid water content of $5-500 \mathrm{~g}-\mathrm{m}^{-2}$.

In order to compute shortwave spectral radiances from the spectral radiant exitances which were based on the two-stream approximation, spectral bidirectional models of four surface scenes and two clouds $\left(h_{c l}=3 \mathrm{~km}\right.$ and $9 \mathrm{~km}$.) by Stuhlmann et al. (1985) were used, which are based on an iterative scheme by Raschke. (1971). The calculations by the 2-stream approximations used in the present study are based on the same atmospheric input data. The computations by Stuhlmann et al. (1985) treat the lower boundary as Lambertian except for water surface, for which the reflection is considered to be partly diffuse and partly specular. The specular part is computed from Fresnel's formula, and the surface is assumed to be wavy.

\section{B. Scene Description}

Atmospheric profiles of temperature and humidity were taken from MCClatchey et al. (1977) for five climatological conditions: subarctic summer SAS, midlatitude winter MLW, midlatitude summer MLS, subtropic summer 
STR, and tropics TRO. These profiles are shown in figure 5. Table I shows as an example the content of the data set of one atmospheric set of one profile (subtropic summer) for both the SW and the $L W$ range. The aerosol density is described by the visibility parameter $B$. The aerosol profile of McClatchey et al. (1972) is used; for this case, B is $56 \mathrm{~km}$. A value of $B=23 \mathrm{~km}$ was also used, corresponding to an increased aerosol density. In addition to the surface temperature of the model, surface temperatures of Tmodel-c and $T_{\text {model }}+c$ were used in the radiance computations. This provides a simulation of the effect of diurnal cycle on the radiances. The change $C$ was taken to be $4^{\circ} \mathrm{K}$ for SAS, MLS and MLW and to he $6^{\circ} \mathrm{K}$ for STR and TRO. All surface scenes are sorted into four classes according to the ERBE classifications: ocean, land, ice/snow, desert.

Spectral radiances were computed for each angular bin for each model type listed in Table II. Thus, for each surface type there is a range of cloud heights and optical thicknesses, in addition to. the three surface temperatures. The set of spectral radiances thus computed constitutes the "level I" data in figure 4. Given the spectral response of various satellite instruments, the resulting measurements of these scenes can be computed by use of equation (2).

\section{C. Spectral Responses of Spacecraft Instruments}

The spectral response of a satellite radiometer to incoming radiation is determined by its optics and the nature of the sensing element. For the total channel of the ERRE scanning radiometer, a Cassegrain telescope consisting of two front surface aluminum mirrors focuses the radiation onto a bolometer, which measures the total radiation impinging on it. The spectral response of this channel is thus the product of the spectral reflectivity of each of the mirrors and the spectral absorbtivity of the coating of the 
bolometer, and was shown in figure 1. The shortwave channel is the same, except for a suprasil filter in front of the detector, which effectively blocks longwave radiation but passes shortwave radiation. The longwave channel has a diamond filter in front of the detector, which blocks shortwave radiation but passes longwave radiation. The spectral response of each of these two channels is that of the total channel times the spectral transmission of the filter, and was shown also in figure 1.

Because data from the ERB scanning radiometer on the Nimbus 6 and 7 spacecraft and the Advanced Very High Resolution Radiometer AVHRR ahoard the NOAA 9 spacecraft are used, it is necessary to consider the spectral responses of these instruments. The shortwave and longwave channels of the ERB scanning radiometer on the Nimbus 6 and 7 spacecraft have practically the same spectral responses as those of ERBE. Data from channels 1, 2 and 4 of the AVHRR are also used, and their spectral responses are shown in figure 6.

The measurement for a given radiometer channel was thus computed by use of its spectral response and the spectral radiance of the scene in equation (2). These simulated measurements constitute the level II data for this study.

III D. Means and Variances of Filtered Radiances

For each scene type, spectral radiances and simulated measurements were computed for each of three surface temperatures and a range of cloud top heights and optical thicknesses for each viewing zenith, solar zenith and azimuth angle, as shown in Table II. For each scene type and angular bin, average radiances, standard deviations and correlation coefficients between the shortwave and longwave measurements were computed. These data, which are called the level III data set, are the kind which are required for in- 
put to the scene identification algorithm for ERBE data processing.

Typical results for the ERBE scanning radiometer are shown in figures $7 \mathrm{a}$ and $7 \mathrm{~b}$, respectively. Average shortwave and longwave channel simulated measurements and their standard deviations are shown versus viewing angle bins in the backscatter direction $\left(150^{\circ}<\phi<180^{\circ}\right)$ and solar zenith angle in the range 32.5 to $37.5^{\circ}$. The scene is tropical ocean for clear and with high and medium height clouds. The clear ocean scene shows the expected limb darkening in the longwave measurement and limb brightening in the shortwave measurement. In the longwave case the radiances of the high cold cloud (HK) increase with increasing $\theta_{v}$ because the atmospheric effects superimpose the low cloud temperatures. For the same reason the shortwave radiances decrease at high viewing zenith bins. The simulation of the sun glint is another feature of the applied bidirectional models. $\left(0^{\circ}<\phi<9^{\circ}\right)$.

The atmospheric temperature and humidity profiles (MC Clatchey et al., 1977) which were used for the longwave radiance computation do not consider the surface types which are used by the ERBE scene identification algorithm. For the present study, the effect of surface type on the longwave measurement is accounted for by empirically determined factors, which are obtained from mean climatological LW exitance data. Thus, the longwave radiance for an atmospheric profile $p$ and scene ss is computed by

where

$$
\left.L_{p} L W_{(} \theta_{v}, s s\right)=L_{p} L W\left(\theta_{v}\right) \times f_{p}(s s)
$$

$$
\begin{gathered}
f_{p}(s s)=\frac{\bar{M} *\left(t_{p}, x_{p}\right)}{2 \pi \int_{0}^{\pi / 2}\left[L W_{\left(\theta_{v}\right)} \sin \theta_{v} \cos \theta_{v} d \theta_{v}\right.} \\
\text { ss = surface scene (ocean, land, ice/snow, desert) } \\
p=\text { atmospheric profile (subarctic winter,..., tropic) }
\end{gathered}
$$

$M \star\left(t_{p}, x_{p}\right)$ is a mean value of all latitude bands $\left(x_{p}\right)$ and the season $\left(t_{p}\right)$, which corresponds with one of the five available atmospheric model profiles. The factor $f_{p}(s s)$ is mainly in the range .9 to 1.1 . 
IV. APPLICATION OF THEORY TO ERBE SCENES.

Simulated measurements were computed for the ERBE SW and LW scanner channels using the spectral responses for these channels together with the computed spectral radiances. The means, variances and correlation coefficients were computed for these simulated measurements. The 1-sigma ellipses are shown in the LW-SW plane in Figure 8. The locations of the centers of the ellipses in this plane are given by the means and their sizes and orientations are determined by the correlation coefficients and variances. Also shown in this figure are real ERBE measurements, with plotting symbols indicating scene types as determined by the ERBE scene identification algorithm. The area is located at the Pacific west of California and Central America, for April 4, 1985. Here only "clear ocean" and "overcast" scenes were chosen.

The ERBE determined overcast scenes are in good agreement with the model "high clouds", with a cluster near the clouds with medium optical thickness. This result is also confirmed by AVHRR data, as will be discussed later (see VB). On the other side the "clear-ocean" pixel cluster is "cooler" and a little "brighter" than the pixel average. This might be due to the large ERBE pixel size (44 km $\star 65 \mathrm{~km}$ at nadir), which most probably leads to cloud contamination. In fact an ERBE scene is denoted as "clear" when the pixels contain less than $5 \%$ cloud, which of course increases the SW and decreases the $L W$ radiance.

Another obvious reason for possible disagreements is the necessary limitation in model input data (albedo models, visibilities, surface temperatures, cloud parameters, atmospheric models). This modifies the mean radiances and diminishes their variances. In spite of this, the data set provides agreement which is good enough that the present method is seen to 
provide an acceptable tool for scene identification.

One conjectures that the data points between the "clear" and "overcast" may be due to high thin and high medium thick clouds. However, they may also be due to partly filled fields of view, or partly cloudy scenes. Use of higher resolution data such as AVHRR imagery is necessary to distinguish between these alternatives. 
V. ERRE-AVHRR (NOAA-9) SCENE-IN INTERCOMPARISON

VA. Technique

The Advanced Very High Resolution Radiometer (AVHRR) on board the NOAA-9 spacecraft with the ERBE instruments provides an excellent opportunity for an independent check on the scene identification as computed by the ERBE procedures. The smaller field of view of the AVHRR gives more pixels which are clear or cloud filled than does the ERBE scanner. Also, techniques have been developed for the identification of clouds with AVHRR data. In this study, the maximum likelihood estimator (MLE), which is the ERBE scene identification algorithm as discussed earlier, is applied to the AVHRR data to compute scene types to provide a comparison with the ERRE computed scene types. Next, the AVHRR scene types are computed by use of the method of Eyre et al. (1984) and comparisons are made.

Data for these intercomparisons are taken from ERRF $V-5$ data tapes, which contain data from both the ERBE and AVHRR radiometers for selected regions of the Earth, for April 14, 1985. Two test sites were chosen, as indicated on the map in figure 9. The first is located in the Pacific Dcean, west of and Central America, as the spacecraft moved from $4.9^{\circ} \mathrm{N}$, $132.4^{\circ} \mathrm{W}$ to $9.7^{\circ} \mathrm{N}, 133.6^{\circ} \mathrm{W}$, covering approximately region number 1197 of the ERBE $2.5^{\circ} \times 2.5^{\circ}$ grid as the scanning radiometer operates in across-track mode. The second is in the Atlantic Ocean west of Africa but including some coastal areas, as the spacecraft moved from $4.9^{\circ} \mathrm{N}, 4.7^{\circ} \mathrm{W}$ to $9.7^{\circ} \mathrm{N}$, $5.9^{\circ} \mathrm{W}$, covering approximately region number 1219 of the ERBE grid.

The MLE method selects a cloud class for a given pair of measurements. In doing so, it treats each pixel independently to attach one of the a priori modeled scene types, characterized by radiance averages, standard deviations and correlation coefficient. In order to apply this method to 
AVHRR data, these statistics; which we call level III data, must be available for the channels of the AVHRR. The methods described in sections II and III were used to generate this required data base, for the 20 scene types.

The present study uses the simplifying assumption that the scene is either clear or completely cloudy. Arking et al. (1983) mention the impacts of this error and use a "maximal clustering technique" to determine cloud fractions of the field of view of each pixel. A different method more suitable for this study is described later (SVD). Another feature of the MLE method is a quadratic dependence on the standard deviation. Pixels which are much warmer than the modeled clear scene $L W$ value with its normally rather small variance (for example, clear "desert" or "land") could thus be identified as a cloud (significantly higher variance). This "wrap around" is avoided in this study by including some restraints in the MLEprobability computation, as is done in the ERBE scene identification algorithm.

In order to apply the MLE method for scene identification from AVHRR data, a new level II data set suitable for the AVHRR is computed using AVHRR channel 2 and 4 filter functions (NOAA Users' Guide), centered around .9 and $11 . \mu \mathrm{m}$, respectively, to simulate $S W$ and $L W$ measurement pairs. A level III data set for the AVHRR measurements was created which includes average filtered radiances for the same 20 scenes listed in Table II. Channel 2 was chosen instead of channel $1(.55-.75 \mu \mathrm{m})$ because in this channel calibrated radiances of various scenes cover a broader range than the respective radiances of channel 1, e.g., clouds appear brighter and clear scenes appear darker in channel 2 than in channel 1 . This behavior obviously allows a better scene discrimination in the SW-range. 
The counts of channels 2 and 4 were calibrated and transformed into radiances using the calibration coefficients stored on the $V-5$ tape using the procedure described by Rossow et al. (1985). Percent scaled radiances for each channel are computed from the counts using tabulated gains and offsets. The effective solar irradiance for each channel is calculated by integrating the product of the spectral response function for the channel and the solar irradiance, as tabulated by Neckel and Lab (1984). The radiance is then the percent scaled irradiance multiplied by the effective solar irradiance for each channel.

With the geographic information of each pixel, an underlying surface scene is taken from an external data set. The a priori model values of this clear scene and of a variety of model clouds above this surface together with the original AVHRR measurement pair serve as input data in the MLE procedure. The model scene with the highest probability is regarded as the "true" scene for the AVHRR pixel. Uncertainties in this scene determination are due to the presupposition of a Gaussian distribution of the pixel in the SW-LW plane and of course to the fact that the model scene possibly does not fit the reality.

One-sigma ellipses in the LW-SW plane for the level III data set for AVHRR measurements are shown in figure $10 \mathrm{a}$ and $\mathrm{b}$ for tropical ocean, land and clouds. Calibrated LW and SW AVHRR data (channels 2 and 4 ), indicated as dots, are compared with the model scene values as in figure 8 . These data are AVHRR GAC measurements from the NOAA 9 satellite on 14 Apri1, 1985 for the two regions earlier described. The GAC data from the V-5 tapes are much smaller $\left(\sim 12 \mathrm{~km}^{2}\right)$ than the ERBE pixel $(44 \times 65 \mathrm{~km}$, NOAA9). It is seen that the AVHRR data lie in the envelope of 1-sigma ellipses of radiances as computed by radiative transfer. While the first case a) consists of cold 
ocean scenes with various. cloud types, the second test area contains clear land pixels and only a few (warm) ocean pixels. The thin cloud types over land are significantly brighter than the respective clouds over ocean in a) and are in good agreement with the modeled scenes (e.g. scene types 8,9 and 10 in Figure $10 \mathrm{b.})$. The influence of the surface can be neglected for medium thick (scenes 13,14 and 15) and thick (scenes 19,20) clouds. This agreement lends support to acceptance of the identification of these scene types, although as before it is noted that it is assumed that all fields of view are either full or empty.

The following problems occur. The variance of the SW radiances for the ocean is small compared to that for partly cloudy over ocean. As a consequence, the MLE method will tend to identify many scenes as cloud. Also, it is difficult to discriminate between clear ocean or clear land and thin and low clouds, as their radiative effects are small. Fortunately, for this reason the influence of these scene identification errors is small. Finally, many GAC pixels which are classed as clear may well be cloud contaminated.

V. Spatial Colocation of ERRE and AVHRR pixels

In order to compare the scene identification resuits from the AVHRR with those from the ERRE scanner, it is necessary to colocate them spatially. The ERBE scanning radiometers have an optical mask in front of each detector which forms a hexagonal instantaneous field of view with the $3^{\circ}$ width in the scan direction and $4.5^{\circ}$ width in the perpendicular direction. The shape of the ERBE pixel is also modified by the time response of the instrument as it scans. Because of this the shape of the surface area covered by an ERRE pixel is approximated in this study hy superimposing an ellipse and a rectangle with the footprint of the pixel in its center, as shown in figure 11 . The length of the major axis "a" along the scan line is approximated as half 
of the distance of two neighboring pixel-footprints. The minor axis "b" is half of the distance of two neighboring footprints along the nadir and is held constant along the scan 1 ine. With the geometric information of both the ERBE and the AVHRR data the distance of the AVHRR footprint perpendicular to the ERBE scan line " $d$ " and the distance to the edge of the ERBE pixel is determined and compared (figure 11). If $|d|<b^{\prime}$, the AVHRR pixel is regarded as being within the ERRE pixel.

$\underline{v}$ C. Method of Cloudiness netermination

As mentioned above, it is necessary to account for partially cloud-filled AVHRR pixels in order to estimate an average cloudiness of the total area. This was done by applying a technique which makes use of the different behaviour of AVHRR channels 1 and 2 reflectances $\left(r_{1}, r_{2}\right)$. For ocean scenes $r_{1}$ is larger than $r_{2}$ and for 1 and and desert the opposite is the case, which might be due to differing aerosol scattering in the channel 2 range or to the spectral characteristics of the incoming rays. For the high resolution AVHRR-LAC data (1 km²), Kriebel (1986) and Saunders (1986) found that for clear ocean $r 2 / r 1 \sim 0.5$ and for land $r 2 / r 1>1$. The maxima for clouds were centered at 0.9 . This value can vary between 0.7 and 1.0 according to cloud top altitudes as a result of change of ozone and water vapor content above the clouds.

In order to apply this behaviour to identify partly cloudy AVHRR pixels, as a first step one of the 20 pre-defined scene types is attached to each AVHRR pixel with a Maximum Likelihood Estimation method. Also the ratios of the reflectances $r_{2} / r_{1}$ are determined for each pixel and the corresponding scenes are sorted into histograms as shown in figure 12 . Recause of the larger pixel sizes the peaks are not so well determined, which points to a 
probable cloud contamination of the clear scenes. Clear ocean, desert and land scenes are clearly separated from each other and show overlapping areas with the cloud cluster. All pixels with ratios $r_{2} / r_{1}$ in these areas where regarded as cloud contaminated or as not totally cloudy in the case of predefined cloud scenes. Boundaries were chosen for clear, mixed and cloudy scenes depending on the respective condition of the histograms of each test area. Then the AVHRR pixel cloudiness amount $c$ is determined. It is 0 for clear scenes and 1 for totally cloudy pixels and linear interpolated between 0 and 1 if $r_{2} / r_{1}$ is beyond the predefined boundaries (e.g. for an ocean scene scene in figure $12,0<c<1$ if $\left.0.75<r_{2} / r_{1}<0.9\right)$.

As a final step these cloud amounts $c$ of all AVHRR pixels belonging to one ERBE pixel results in a mean percentage cloudiness $\bar{c}$ of the pixel area which is according to the ERBE scheme :

$$
\begin{array}{ll}
0 \%<\bar{c}<5 \%: \text { clear } & , j=1 \\
5 \%<\bar{c}<50 \%: \text { partly cloudy. } & , j=2 \\
50 \%<\bar{c}<95 \%: \text { mostly cloudy }, & j=3 \\
95 \%<\bar{c}<100 \%: \text { overcast } & , j=4
\end{array}
$$

The type of cloudiness is thus computed for each area covered by an ERBE pixel (see Table II). These scenes $j$, here regarded as "truth", can finally be compared with the respective ERBE scenes of the same area.

The ERBE-AVHRR scene intercomparison were done for all pixels of a test area and the results were sorted into matrices as shown in Table III for region 1197. In part (a) of Table III, all intercomparisons are taken into account. In 353 cases the ERBE scene is computed to be clear ocean $(i=1)$ whereas partly cloudy scenes are determined from the AVHRR measurements using the method described above. The example shows clearly some other features that are found. In the clear and partly cloudy cases for AVHRR, there 
are deviations of the ERBE to both more and less cloudiness. A slight tendency for underestimation can be seen at partly cloudy scenes. Yet this feature is very clear at mostly cloudy and overcast scenes, where ERBE for instance determines 687 mostly cloudy scenes that were classed by the AVHRR procedure as overcast.

The matrices in part b) of Table III contain all those intercomparisons with the restriction that a single AVHRR determined cloud type is dominant for each cloud type (c (cloud type) > $50 \%$ ). From the first matrix it is evident that the underestimation is due to thin clouds and in a less degree to low and middle high clouds. On the other hand, there is almost always a fairly good agreement at medium, thick and high clouds. In all cases only very few overestimations of cloudiness from the ERBE are observed. 
VD. Figures of Merit for Intercomparisons

Two methods are chosen as means to compare the matrices of different areas, from now on called probability-matrices $P_{i j}$ according to Smith et a1. (1984). As the first characteristic the sum of the diagonal elements (denoted $-3, \ldots, 3$ ) as shown below are determined and their percentage of the matrix-elements total is plotted as histograms (Figure 13) for the five cases which were studied.

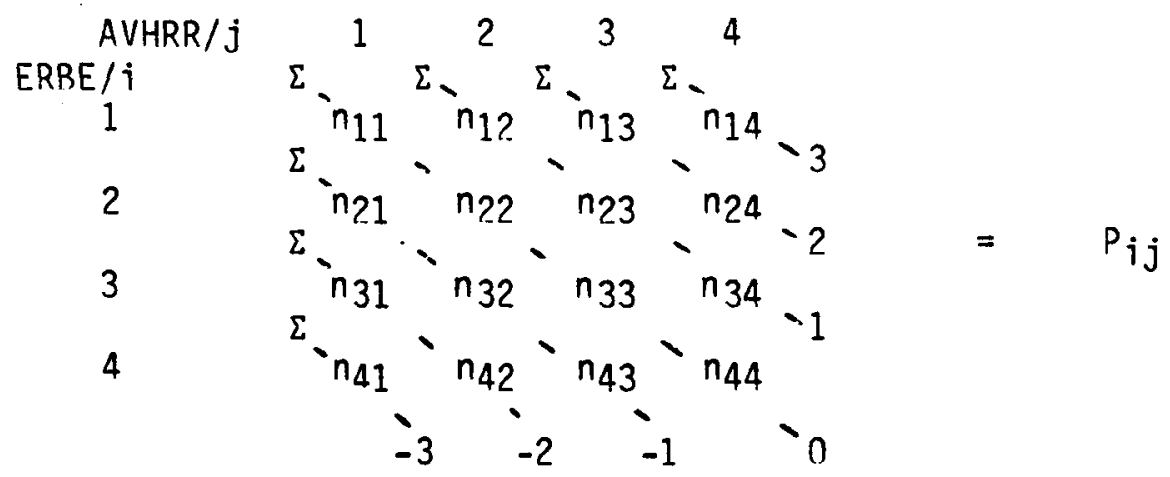

In order to characterize the degree of misinterpretation of scenes a weighting matrix is defined as

$$
\underset{\substack{j=1,4 \\
j=1,4}}{\left(a_{i j}\right.}=\left[\begin{array}{llll}
1 & 2 & 3 & 4 \\
2 & 1 & 2 & 3 \\
3 & 2 & 1 & 2 \\
4 & 3 & 2 & 1
\end{array}\right]
$$

Two more matrices are necessary in order to define a parameter which is a measure for the distribution of the elements within a matrix :

$$
\left(c_{i j}\right)=\left\{\begin{array}{c}
a_{i j}, p_{i j}>0 \\
n, p_{i j}=0
\end{array} \quad \text { and } \quad\left(d_{i j}\right)=\left\{\begin{array}{l}
1, p_{i j}>0 \\
0, p_{i j}=0
\end{array}\right.\right.
$$

Also the following quantities are defined:

$$
\begin{aligned}
& P=\sum_{i, j} P_{i j} \\
& A=\sum_{i, j} P_{i j} a_{i j} \\
& C=\sum_{i, j} c_{i j}
\end{aligned}
$$


$D=\sum_{i, j} d_{i j}$

With these numbers and denoting the number of rows/columns as $n$, the required parameter MM can be defined :

$$
\begin{gathered}
T=\frac{n}{n-1}-\frac{1}{n-1} \frac{A}{P} \quad 1<\frac{A}{P}<n, 0<T<1 \\
S=\frac{n}{n-1}-\frac{1}{n-1} \frac{C}{D} \quad 1<\frac{C}{D}<n, 0<S<1 \\
\text { Def. : MM }=T \sqrt{S}
\end{gathered}
$$

MM stands for "Matrix Moment" and is 1 if all elements are on the middle diagonal (absolut agreement) and 0 if they are located in the lower left and upper right corners (total disagreement). $T(A / P)$ is the normalized average weighting factor of the matrix $P_{i j}$ with the worst result at $A / P=4$. $C / D$ is a factor that takes the distribution of the elements within the matrix into account. Figure 13 demonstrates that for equal values of $A / P$, MM decreases if the matrix-elements are changed toward the corners.

The following example shows two matrices with 14 elements in different positions but with the same ratio $A / P=24 / 14$.

$$
\begin{array}{rrrlll}
4 & 0 & 0 & 0 & A / P=24 / 14 & T=0.762 \\
10 & 0 & 0 & 0 & & \\
0 & 0 & 0 & 0 & C / D=3 / 2=1.5 & S=0.834 \\
0 & 0 & 0 & 0 & & M M=0.696 \\
M & & & & \\
M & & & \\
10 & 0 & 0 & 0 & C / P=24 / 14 & T=0.762 \\
1 & 0 & 0 & 0 & C / D=7 / 3=2.3 \quad \\
0 & 0 & 0 & 0 & S=0.567 \\
3 & 0 & 0 & 0 & & M M=0.574
\end{array}
$$

Although there are more elements on the middle - diagonal at $M_{2} M M$ is lower because of three elements being at the worst position in the lower left corner. 
VE. Discussion of Intercomparison Results

Figure 14 shows histograms of the normalized diagonal sums and the matrix moment MM for several cases. The histograms show similar patterns: MM between is between .65 and .73 in all cases and there are only few strong deviations from the middle diagonal. In four cases there is generally more underestimaion of cloudiness according to the ERBE scene identification method. This underestimation occurred often at thin and low clouds, whereas there is almost always a good agreement at medium, thick and high clouds. Thus ERRE tends to interpret thin clouds in lower levels as clear scenes (at high cloud amounts), or more accurately, the AVHRR scene identification method determines more thin and low clouds in spite of its tendency to denote those pixels as mixed scenes (see VD, $0<c_{\operatorname{mix}}<1$ ).

A significant overestimation of cloudiness is found in only one case (April 14, 1985 for 1219 Atlantic/Northwest Africa). This is the only test area that contains about $30 \%$ clear and cloudy land, desert and coastal scenes according to the a priori ERBE surface scene map. Reasons for this might be the fact that the "land" scenes of this part of Africa are found to be very hot and sometimes as bright as "desert" scenes. Together with the "wrap around" effect of the MLE method this could be one reason for about $20 \%$ of all clear land scenes (AVHRR) being denoted as "partly cloudy" by the ERBE scene method. Finally there are several bright and extremely hot desert scenes determined by the AVHRR in areas the ERBE geotype map has predefined as "coastal" areas. Due to the "wrap around" effect these obviously "clear" scenes are misidentified as partly cloudy and in several cases even as overcast scenes by ERRE.

On April 19, 1985 at the same test area (1219) there are several wrong (overcast) AVHRR scenes found in the sun-glint area of clear ocean where 
the ERBE quotes the scene rightly (according to albedo and surface temperature) as cloud-free ocean. That indicates a reasonable handling of the sun-glint in the ERBE inversion algorithm. This statement is also confirmed by the fact that neglecting all comparisons in the sun-glint area does not improve the results of the probability matrices.

The matrices shown as an example in Table III were also sorted into all combinations of angular bins. It can be seen that misinterpretations of ERBE scenes are at no preferred angular bin, e.g. there are no better intercomparison results in backward than in forward scatter direction. Finally, if the ERBE determines a scene as overcast, it is most probably true because there are only very few overestimates at high cloud amounts (overcast instead of mostly cloudy) for all cases. 
VI. ERROR PROPAGATION IN THE ERRF INVERSION ALGORITHM

VI A. Theoretical Considerations

In this study, the approach of Smith et a1. (1984) will be used. The total error of the radiant exitance for each pixel is the sum of two basic components. The error in scene identification results in discrete changes in the spectral correction and bidirectional functions (Fig. 15), or branching in the computational flow. The second error component contains term errors (bidirectional function- and spectral factor-variations, instrument noise) and can be treated in a linear manner. In this study some first estimates for the errors due to scene misidentification will be presented.

Let a scene which is in truth type " $j$ " be identified as type " $i$ ". Then the expected error in the estimate of radiant exitances $M$ is defined as

$$
E\left(\varepsilon_{M i}\right)=\Sigma_{j} \varepsilon_{M i j} P_{i j}
$$

where $P_{i j}$ is the probability that a scene is in truth type $j$ and that it is identified as type $i$. This error, if nonzero, represents a bias in the analysis of the data. Assuming that this bias is zero the variance of this error is

$$
\left.V\left(\varepsilon_{M i j}\right)=\sum_{j} \varepsilon_{M i j}^{2} P_{i j}-\Gamma \sum_{j} \varepsilon_{M i j} P_{i j}\right]^{2}=\sum_{j} \varepsilon_{M i j}^{2} P_{i j}
$$

The probability $P_{i j}$ is the product of the conditional probabilities that a scene is in truth type $j$ and that it is identified as type $i$. As such, it can be written as

$$
P_{i j}=P_{j} \int_{R_{j}} \pi_{j}(x) d x
$$

where $x$ is a two dimensional radiance space ( $\left.L^{S W}, L^{1 w}\right)$. The probability of occurence of various scene classes $P_{j}$ represents the statistical makeup of the cloud field, and $\Pi_{j}(x)$ is the probability that if the scene is of type $j$ that it will have value $x$. The domain of integration is the part of the $x$ or $\left(L^{S W}, L^{1 W}\right)$ plane in which the scene identification algorithm identifies 
the scene to he of type $i$. The probability distribution $\pi_{j}(x)$ will he considered to he a normalized prohability distribution of the measured radiance pair $\left(L^{S W}, L^{T W}\right)$ for scene type $j$ and can be approximated as

$$
\begin{gathered}
\Pi_{j}(x)=\left(\sigma_{S W} \sigma_{1 w} 2 \pi \sqrt{1-\rho^{2}}\right)^{-1} e^{-0} \\
0=\frac{1}{2\left(1-\rho^{2}\right)}\left(\frac{u^{2}}{\sigma_{S W}^{2}}+\frac{v^{2}}{\sigma_{T W}^{2}}-2 \rho \frac{u v}{\sigma_{S W} \sigma_{T W}}\right) \\
u=L S W-L S W \\
v=L^{T w}-L^{T w}
\end{gathered}
$$

The means, variances and correlation coefficients of shortwave and longwave radiances are considered to be known for each scene type $j$. The integration is over the region which is defined by the scene identification algorithm using equation (8) as being scene type $i$, indicated in figure 16 by the cross-hatched area. These scene areas are delineated by elitipses which are given by the equation

$$
P_{j} \Pi_{i}=P_{j} \Pi_{j}
$$

Because the Gauss distribution for scene classes may be an unrealistic assumption for points well away from the centroid of each scene type, it is desirable to use the $P_{i j}$ as computed in the last chapter. The AVHRR scene type is again considered to be truth $j$.

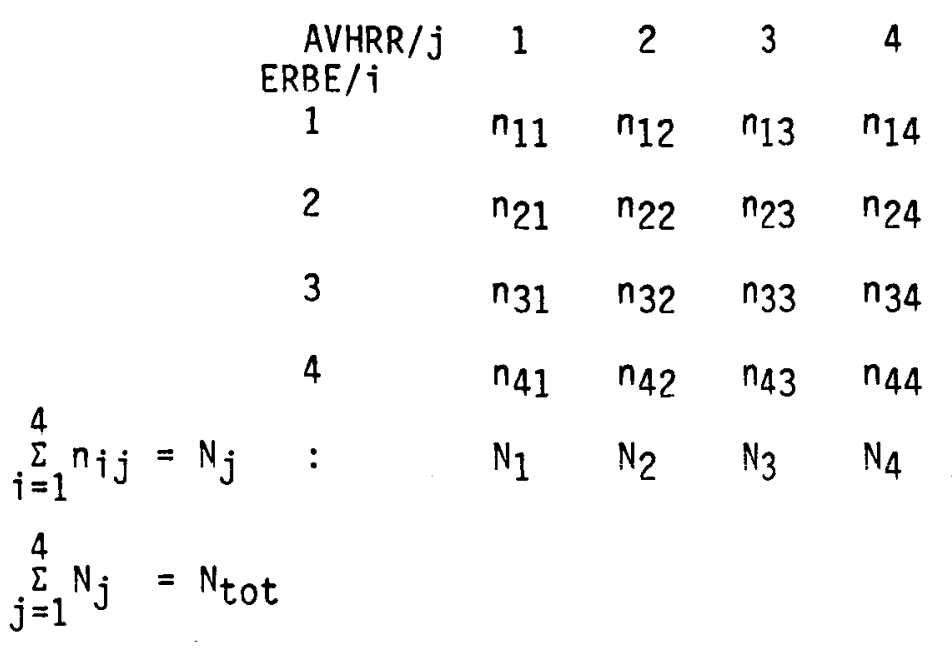


The portion of scene class $j$ identified as $j$ (the cross-hatched area in figure 16 ) is thus evaluated empirically rather than by the integral expression in equation (7):

$$
P_{i j}=\frac{N_{j}}{N_{\text {tot }}} \times \frac{n_{i j}}{N_{j}}=\frac{n_{i j}}{N_{\text {tot }}}
$$

The ratio of $N_{j}$ and the total number of pixels in the test area $N_{t o t}$ is an empirical evaluation of $P_{j}$, and the ratio of $n_{j j}$ to $N_{j}$ is an empirical evaluation of $P_{i j}$. The $P_{i j}$ are only valid for a certain area and based on a "truth" which of course is an estimate, based on many uncertainties, by itself.

If the scene is jdentified as type $i$ the shortwave and longwave radiant exitances at TOA, expressed as components of a two-dimensional vector, are estimated as

$$
\underline{M}_{j}=\pi Q_{j} C_{j} \underline{m}
$$

whereas the correct exitance for scene class $j$ would be

$$
\underline{M}_{j}=\pi Q_{j} C_{j} \underline{m}
$$

$Q$ is a $2 \times 2$ diagonal matrix with $1 / R$ on the diagonal for the shortwaveand longwave component, respectively. $C$ is the $2 \times 3$ spectral correction matrix and $m$ a vector of ERBE shortwave, longwave and total channel radiances.

The error $\underline{\varepsilon} M j j$ in the estimate of radiant exitance due to scene errors is then

$$
\underline{\varepsilon} M_{i j}=\pi\left(Q_{j}-Q_{j}\right) C_{j} \underline{m}+\pi Q_{j}\left(C_{j}-C_{j}\right) m
$$

The covariance matrix for $\underline{\varepsilon}_{M i j}$ is given by (analogous to (6)): 


$$
\begin{aligned}
& \operatorname{cov}\left(\varepsilon_{M j}(\text { scene })\right)= \\
& \left.\pi^{2} \sum_{j=1}^{4} P_{i j}\left(0_{i}-0_{j}\right) \Gamma_{L_{i}} \underline{L}_{i}^{T}+\operatorname{cov}\left(\underline{L}_{i}\right)\right]\left(0_{i}^{T}-0_{j}^{T}\right) \\
& +\pi^{2} \sum_{j=1}^{4} p_{i j} 0_{j}\left(C_{i}-C_{j}\right)\left[\underline{m}_{i} \underline{m}_{i}^{T}+\operatorname{cov}\left(\underline{m}_{i}\right)\right]\left(C_{i}^{T}-C_{j}^{T}\right) 0_{j}^{T} \\
& +\pi^{2} \sum_{j=1}^{4} p_{i j}\left(K_{i j}+K_{i j}\right)
\end{aligned}
$$

Here the approximation is made that

$$
P_{j} \int_{R_{j}} \Pi_{j}(x) d x \underline{m} \underline{m}^{\top}=P_{i j}\left(\bar{m}_{i} \underline{m}_{i}^{T}+\operatorname{cov}\left(\underline{m}_{j}\right)\right)
$$

The second moment of the distribution is in this way evaluated by use of the a priori mean values of the identified scene radiances.

The first term on the right hand side of equation (14) is the error variance due to bidirectional modeling error, the second term is the error variance due to spectral modeling error, both as results of scene-identifi= cation error, and the remaining parts are coupling terms between these two effects.

\section{B. Preliminary Estimates of Exitance Errors}

The shortwave and longwave exitance standard deviations are the squareroots of the diagonal elements of the covariance matrices. For each pixel, the percentage deviation can be calculated:

$$
p_{i}=\frac{\sigma_{i}}{M_{i}} \cdot 100 \quad[\%]
$$

Figures 17 and 18 show preliminary results of these errors for the ERRE scenes in region 1197 on April 4, 1985. The probability matrix for this case is:

$$
P_{i j}=\left[\begin{array}{cccc}
0.127 & 0.041 & 0.001 & 0 \\
0.059 & 0.140 & 0.038 & 0.001 \\
0.011 & 0.065 & 0.130 & 0.082 \\
0 & 0.001 & 0.007 & 0.297
\end{array}\right]
$$


Because of the low likelihood of underestimating an overcast scene the shortwave errors for this scene type form the minimum areas in figure 17. Error maxima are almost always connected with mostly cloudy and sometimes partly cloudy scenes. Reasons for this are the great amount of variability in cloud types, altitudes and total amount ( $5-50$ and $50-95 \%)$ which causes the radiometer signal $m$ and finally the radiance estimate $M$. The extremely high deviations, indicated with " $H$ " in figure 17 are only very scattered and mostly caused by overestimation of cloudiness when compared with corresponding "true" scenes. Table IV summarizes these results for one case. Errors are averaged over three parts of an ERBE scan line along the flight track. Although these values are based on a limited statistical sample and only valid for a certain area, they demonstrate some typical features. Obviously the longwave errors are negligible for all scene classes and also the shortwave deviations are very low for overcast scenes. For all scenes there is an increasing absolute error.in forward scatter direction but only for clear ocean this is also the case for the percentage error. For all cloudy scene types the shortwave exitances are more increasing than the absolute errors along the scan-line toward the forward scattering of the sun 1ight, and therefore the percentage maxima are in the nadir region. This clearly indicates that the shortwave radiant exitance error due to scene identification error depends on the direction of the reflected sunlight for a clear ocean surface more than for cloudy scenes, because of the sun-glint effect over the ocean. 
VII. CONCLUSIONS

The scene identification probability matrices quoting the probability that a scene which is in truth type $j$ is identified as type $i$ are required for the evaluation of data analysis errors. This paper presents empirical evaluations of these matrices by checking the accuracy of the ERRE scene identification algorithm. This procedure attaches a scene (clear surfaces, partly and mostly cloudy above these surfaces and ocercast) to a shortwavelongwave measurement pair with a maximum likelihood estimation method. In order to perform an intercomparison of these scenes with an independent set of satellite data, a scene identification procedure is developed for AVHRR SW and LW data by using two radiative transfer models. These AVHRR GAC pixels are then attached to corresponding ERBE pixels and the results are sorted into scene or probability matrices.

These scene-type intercomparisons show that there generally is a higher tendency for underestimation of cloudiness over ocean'at high cloud amounts, e.g. mostly cloudy instead of overcast and partly cloudy instead of mostly cloudy, of the ERRE scene identification relative to the AVHRR results. This is mostly caused by thin or low clouds, which on the other hand has only a small impact on the results hecause of the similarity of the respective bidirectional functions. Another error source is the so-called "wrap around" effect in the maximum likelihood method which causes cloudiness overestimation over hot 1 and and coastal scenes.

Preliminary estimates of the errors in radiant exitances which are due to scene misidentification demonstrates the high dependency on the probability matrices. While the longwave error can generally be neglected the shortwave deviations have reached maximum values of more than $12 \%$ of the respective exitances. 
VIII. FUTURE RESEARCH REQUIRED

It is planned as a continuation of this study to develop a nightime scene-identification method for AVHRR data using only the longwave channels 3 (centered at $3.7 \mu \mathrm{m}), 4$ (centered at $11 \mu \mathrm{m}$ ) and 5 (around $12 \cdot \mathrm{m}$ ). The difference in brightness temperatures of channel 3 and 4 for example enables us to detect low clouds, fog or thin cirrus. This feature is described and used for image processing by Eyre et al. (1984) or for retrieval of cloudfree pixels at night by Saunders (1986).

In order to estimate the total pixel error, the uncertainties in the signal $\left(m_{s w}, m_{1}\right)$, the filter factors $(C)$ and the bidirectional functions $(R)$ must be taken into account. These error sources propagate in the inversion algorithm in a linear manner. Further theoretical studies are necessary to estimate the variances of $R_{S W}$ and $R_{T W}$. Also, the analysis of Smith et al. (1984) should be extended to the case of the expected error of a specific measurement. 


\section{REFERENCES}

Arking, A. and J. D. Childs, 1983: Extraction of cloud cover parameters from multispectral satellite measurements. Proceedings of the Fifth Conference on Atmospheric Radiation of the American Meteorological Society, Baltimore, Maryland, 0ct. 31-Nov. 4 .

Avis, L.M.; R. N. Green; J. T. Suttles and S. K. Gupta, 1984: A robust pseudoinverse spectral filter applied to the Earth Radiation Experiment (ERBE) scanning channels, NASA TM-85781, 33 p.

Barkstrom, B. R. and G. L. Smith, 1986: The Earth Radiation Budget Experiment (ERBE): Science and implementation. Rev. Geophys. and Space Physics, 24, 379-390.

Coakley, J.A., Jr. and F. P. Bretherton, 1982: Cloud cover from high resolution scanner data: Detecting and allowing for partially filled fields of view. J. Geophs. Res., 87, C7, 4917-4932, June 20.

Eyre, J. R., J. L. Brownscombe and R. J. Allain, 1984: Detection of fog at nightusing advanced very high resolution radiometer (AVHRR) imagery, Meteorological Magazine, 113, 266-271.

Jacobowitz, H., H. V. Soule, H. Lee Kyle, and F. B. House, 1984: The Earth Radiation Budget (ERB) Experiment: An Overview. J. Geophy. Res., 89, $04,5021-5038$.

Kerschgens, M. E., E. Raschke and U. Reuter, 1976: The absorption of solar radiation in model atmospheres, Contrib. to Atmos. Phys., 49, 81-97. Kriebel, K. T., 1986: Optical properties of clouds from AVHRR/2 data, Proceedings of the Sixth Conference on Atmospheric Radiation of the American Meteorological Society, Williamsburg, Virginia, May 13-16, 78-80.

- Llewellyn-Jones, D. T., P. J. Minnett, R. W. Saunders and A. M. Zavody, 
1984: Satellite multichannel infrared measurements of sea surface temperature of the northeast Atlantic Ocean using AVHRR/2, Quart. J. Roy. Met. Soc., 110, 613-631.

Major, G., 1976: Effects of gases, aerosols and clouds on the atmospheric absorption of solar radiation. Beitraege zur Physik der Atmosphaere, 49, $216-221$.

McClatchey, R. A., H.-J. Bolle and K. Y. Kondratiev, 1977: Report on the IAMAP Radiation Commission Working Group on a standard atmosphere, Seattle, Washington.

Neckel, H. and D. Labs, 1984: The solar radiation between 3300 and $12500 \AA$, Solar Physics, 90, 205-258.

Raschke, E. and W.R. Bandeen, 1970: The radiation balance of the planet Earth from radiation measurements of the satellite Nimbus 2. J. Appl. Meteor., 9, $215-238$.

Raschke, E., 1972: Die Strahlungsbilanz des Systems Erde-Atmosphaere - Neuere Ergebnisse von Satellitenmessungen. Zeitschrift fuer Geophysik, 38 , $967-1000$.

Raschke, E., 1972: Multiple scattering calculations of the transfer of solar radiation in an atmosphere-ocean system, Contrib. to Atmos. Phys., 45 , $1-19$

Raschke, E.; T.H. Vonder Haar; W.R. Bandeen and M. Pasternak, 1973: The Annual Radiation Balance of the Earth-Atmosphere System during 19691970 from Nimbus 3 Measurements. J. Atmos. Sci., 30, 341-364.

Rossow, W. B., E. Kinsella, A. Wolf and L. Garder, 1985: ISSCP, Description of reduced radiance data, WMO/ICSU.

Saunders, R. W., 1986: Retrieval of surface and cloud parameters from AVHRR/2 data, Proceedings of the Sixth Conference on Atmospheric 
Radiation of the American Meteorological Society, Williamsburg, Virginia, May $13-16,81-84$

Schmetz, J. and E. Raschke, 1981: An approximate computation of infrared radiative fluxes in a cloudy atmosphere, PAGEOPH, 119, 248-258. Schmetz, J., E. Raschke and H. Fimpel, 1981: solar and thermal radiation in maritime stratocumulus clouds, Contrib. to Atmos. Phys., 54, 442-452. Smith, G. L., R. N. Green, E. Raschke, L. M. Avis, J. T. Suttles, B. A. Wielicki, and R. Davies, 1986: Inversion methods for satellite studies of the Earth's radiation budget: Development of algorithms for the ERBE mission. Rev. Geophys. and Space Phys, 24, 407-421.

Smith, G. L., L. M. Avis, R. N. Green and B. A. Wielicki, 1984: Method for estimating errors for radiation budget based on a scanning radiometer, Proceedings of Conference on Satellite Meteorology/Remote Sensing and Applications, Clearwater Beach, Florida, June 25-29.

Smith, W.L., D. T. Hilleary, H. Jacobowitz, H. B. Howell, J. R. Hickey and A. J. Drummond, 1977: Nimbus-6 Earth Radiation Budget Experiment. Appl. Opt., 16, 306-318.

Stuhlmann, R., P. Minnis and G. L. Smith, 1985: Cloud bidirectional reflectance functions: A comparison of experimental and theoretical results. App1. Opt., 24, 396-401.

Taylor, V. R. and L.L. Stowe, 1983: Reflectance characteristics of uniform Earth and cloud surfaces derived from Nimbus-7 ERB, J. Geophys. Res., 89, no. D4, 4987-4996.

Vemury, S.K.; L.L. Stowe and H. Jacobowitz,1984: Sample size and scene identification (cloud): Effect on albedo, J. Geophys. Res., 89, no. D4, 5345Vemury, S. K., 1985: Validation of the ERBE scanner scene methodology: Analysis with Nimbus 7 ERB data, NASA Contractor Report 172596. 
$X$. ACKNOWLEDGEMENTS

The authors wish to thank Mrs. Mary Alice Woerner of Langley Research Center for the benefit of her expertise and help with the computer system and data management problems, and Dr. Shashi Gupta of PRC/Kentron Corp. for the use of his software for reading the V-5 ERBE validation data tape. Also, the authors wish to thank Professor Ehrhard Raschke of the Institute for Geophysics and Meteorology of the University of Cologne for the suggestion of the original idea of this work and for making it possible for FJD to engage in this research. 
XI. LIST OF FIGURES

1. Spectral response of ERBE scanning radiometers channels

(Smith et a1.., 1986).

2. Inversion processing steps (Smith et a1., 1986).

3. Schematic of maximum likelihood estimate (Smith et al., 1986).

4. Schematic flow of simulation approach.

5. Vertical profiles of temperature and relative humidity for five atmospheric models (McClatchey, 1983) used in this study.

6. Spectral responses of NOAA 9 AVHRR channels 1,2 and 4 (from NOAA Operational Satellite User's Manual).

7. Computed means and standard deviations for filtered radiances as a function of viewing zenith angle for

a) ERBE shortwave channel and b) longwave channel.

8. Bispectral distribution of ERBE measurements aboard NOAA 9 spacecraft and modeled one-sigma ellipses within for a sample case. For Apri1 4, 1985, area 1197 (see text).

9. Location of orbit tracks for data periods.

10. Bispectral distribution of AVHRR measurements aboard NOAA 9 spacecraft and modeled one-sigma ellipses within for a sample case.

a) for Apri1 4, 1985, area 1197

b) for April 14, 1985, area 1219 (see text).

11. Geometry of mapping AVHRR pixels into ERBE pixels.

12. Histogram of ratio Channel 2/Channel 1 for AVHRR data for differing scene types.

13. Dependence of matrix moment $M M$ on $A / P$ and $C / D$.

14. Histograms of sums of matrix diagonals

15. Branching of computational flow due to scene identification.

16. Schematic of scene identification probability computation.

17. Standard deviation of shortwave radiant exitance errors due to scene identification errors. 


\section{TABLE I}

Example of one level I data set

Mode 1: Subtropic - summer

a) Range: $0.2-3.28 \cdot \mathrm{m} \quad$ (Shortwave)

Spectral fluxes of each scene for all combinations of

- 37 spectral intervals

- 16 sun zenith angles $\left(0, \ldots, 72.5^{\circ}\right)$

- 15 cloud top heights $(1, \ldots, 15 \mathrm{~km})$

- 12 optical depths $(1, \ldots, 100)$

- cloud-free case

Surface scenes

Albedo mode 1

ocean

ocean

limestone, light soil

dry meadow, grass

savannah

sand

sand

wasteland, subtropic low

wasteland, subtropic middle

wasteland, subtropic high

wasteland, subtropic high

$$
\begin{gathered}
\text { visibility }(\mathrm{km}) \quad \text { scene } \\
\text { classification }
\end{gathered}
$$

56

23

56

56

56

56

23

56

56

56

23 ocean

ocean

desert

1 and

land

desert desert

1 and

desert desert desert

b) $4.09-400 \mu \mathrm{m} \quad$ (longwave)

Spectral fluxes for all combinations of

- 51 spectral intervals

- 3 surface temperatures $\left(T_{\text {model }}-c, T_{\text {model }}, T_{\text {mode }}+c\right.$ )

- 9 viewing zenith angles $\left(0, \ldots, 80^{\circ}\right)$

- 15 cloud top heights $(1, \ldots, 15 \mathrm{~km})$

- 9 optical depths $(1,2,4,6,8,10,12.5,15,20)$ at $\zeta=.55 \mu \mathrm{m}$.

- cloud-free case 
TABLE II •

MODEL SCENES

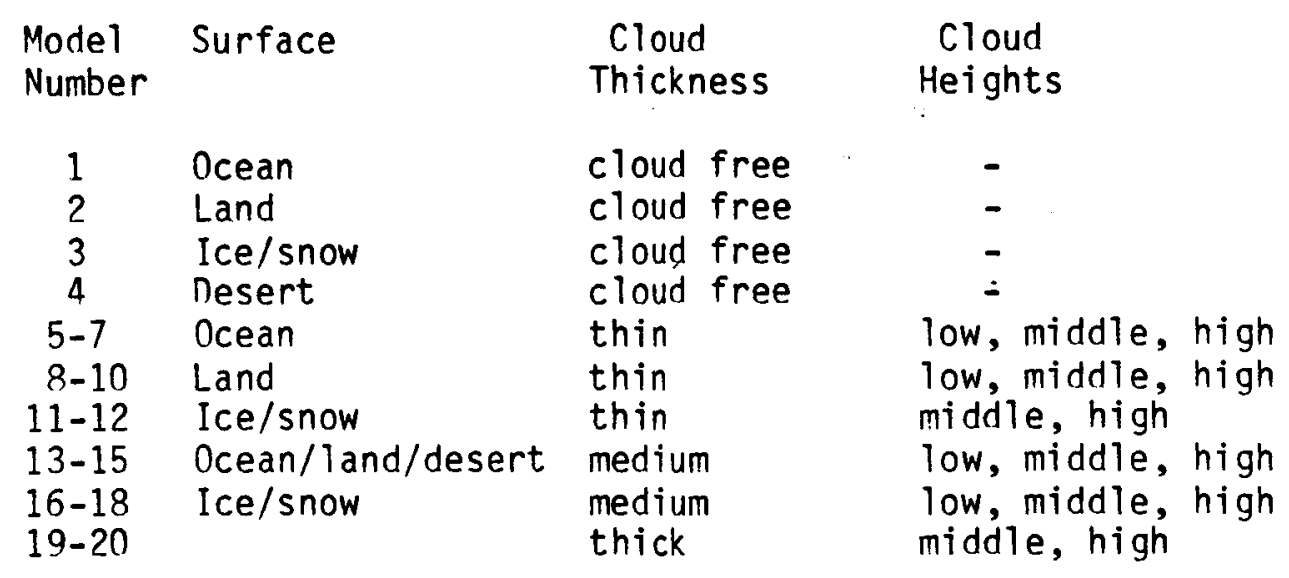

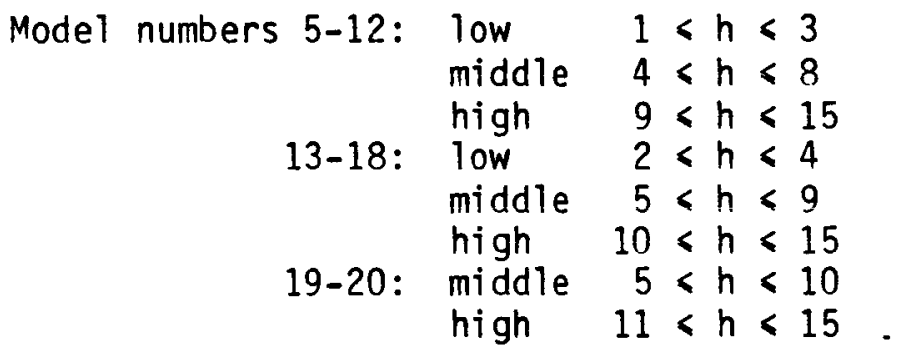

Cloud thickness Optical thickness

$\begin{array}{ll}\text { thin } & 1<\tau<6 \\ \text { medium } & 8<\tau<20 \\ \text { thick } & 30<\tau<100\end{array}$

Angular bins, degrees:

$$
\begin{aligned}
& \theta_{0}: 0-2.5,2.5-7.5,7.5-12.5, \ldots, 67.5-72.5 . \\
& \theta_{v}: 0-10, \ldots, 70-80 . \\
& \phi: 0-9,9-30,30-60,150-180 .
\end{aligned}
$$


Table III

Scene matrices for :

region $1197 \quad$ Apri1 14, 1985

AVHRR : $j=1,2,3,4 \quad$ scene type from averaged cloudiness $c$

ERBE : $i=1,2,3,4 \quad " \quad$ " FRRE scene $i . d$.

a) all pixel intercomparison :

$\begin{array}{crrrr}\begin{array}{c}\text { AVHRR } / j \\ \text { ERBE } / i\end{array} & 1 & 2 & 3 & 4 \\ 1 & 543 & 353 & 74 & 0 \\ 2 & 177 & 688 & 390 & 25 \\ 3 & 32 & 283 & 803 & 687 \\ 4 & 0 & 1 & 45 & 811\end{array}$

b) more than $50 \%$ of all AVHRR scenes covering one ERBE pixel (mostly cloudy, overcast) are :

1. thin clouds

$\begin{array}{llrrr}\text { AVHRR } / j & 1 & 2 & 3 & 4 \\ \text { ERBE } / i & & & & \\ 1 & 0 & 0 & 1 & 0 \\ 2 & 0 & 0 & 167 & 25 \\ 3 & 0 & 0 & 557 & 602 \\ 4 & 0 & 0 & 22 & 354\end{array}$

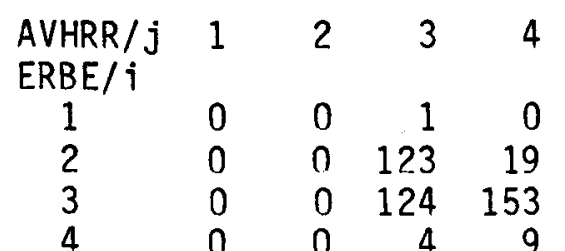

\section{Iow clouds}

2. medium clouds

$\begin{array}{llll}1 & 2 & 3 & 4\end{array}$

$\begin{array}{llll}0 & 0 & 0 & 0\end{array}$

$0 \begin{array}{lll}0 & 1 & 0\end{array}$

$\begin{array}{rrrr}0 & 0 & 5 & 72 \\ 0 & 0 & 1 & 241\end{array}$

\section{3.thick clouds}

$\begin{array}{llll}1 & 2 & 3 & 4\end{array}$

$\begin{array}{llll}0 & 0 & 0 & 0\end{array}$

$\begin{array}{llll}0 & 0 & 0 & 0\end{array}$

$\begin{array}{llll}0 & 0 & 0 & 3\end{array}$

$\begin{array}{llll}0 & 0 & 2 & 161\end{array}$

$$
\text { 5. mid.high clouds }
$$

6. high clouds

$\begin{array}{rrrrrrrr}1 & 2 & 3 & 4 & 1 & 2 & 3 & 4 \\ 0 & 0 & 0 & 0 & 0 & 0 & 0 & 0 \\ 0 & 0 & 13 & 4 & 0 & 0 & 3 & 0 \\ 0 & 0 & 113 & 246 & 0 & 0 & 104 & 238 \\ 0 & 0 & 7 & 80 & 0 & 0 & 15 & 528\end{array}$


Table IV: Averaged standard deviations

$$
p_{i}=1 / N_{\text {pixel }} \stackrel{\sum_{M i}}{\sum_{i} / 100}
$$

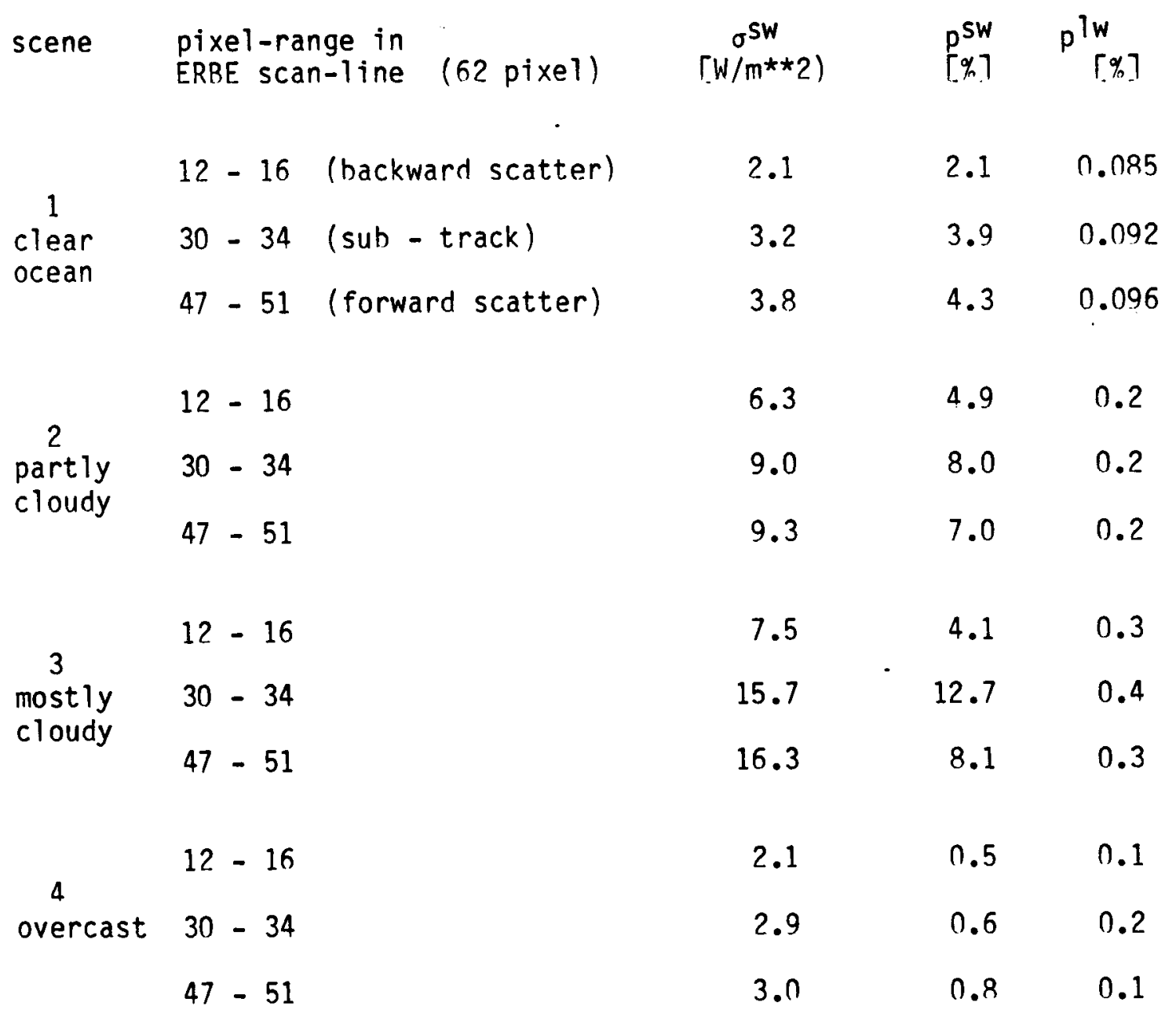




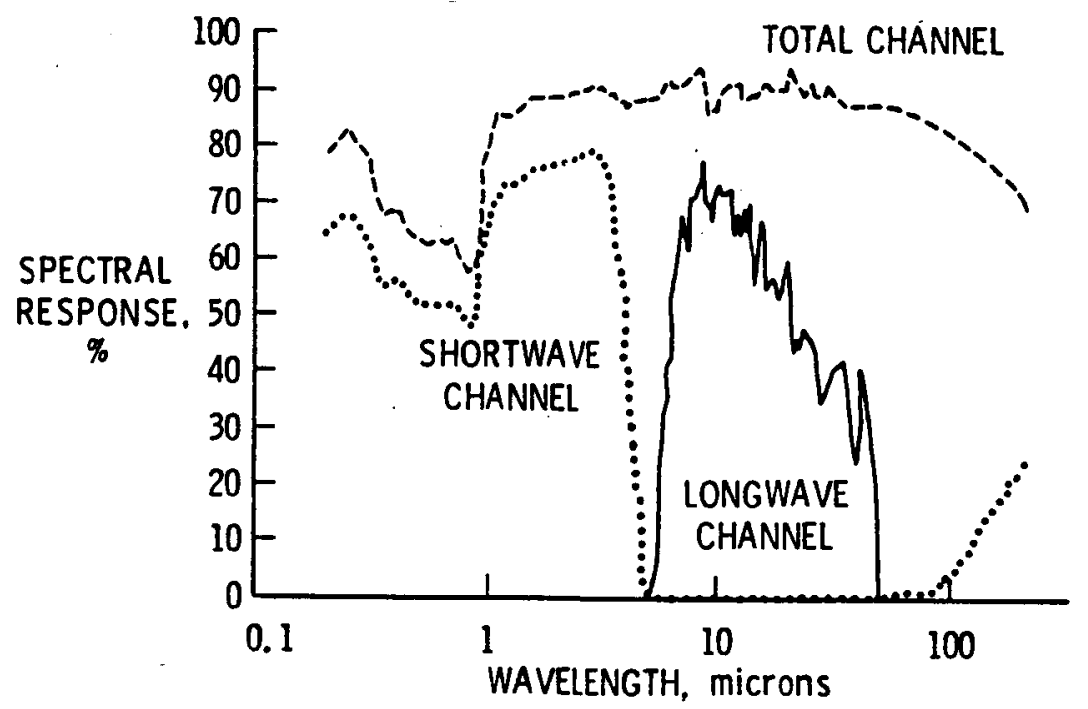

1. Spectral response of ERBE scanning radiometers channels (Smith et al., 1986). 


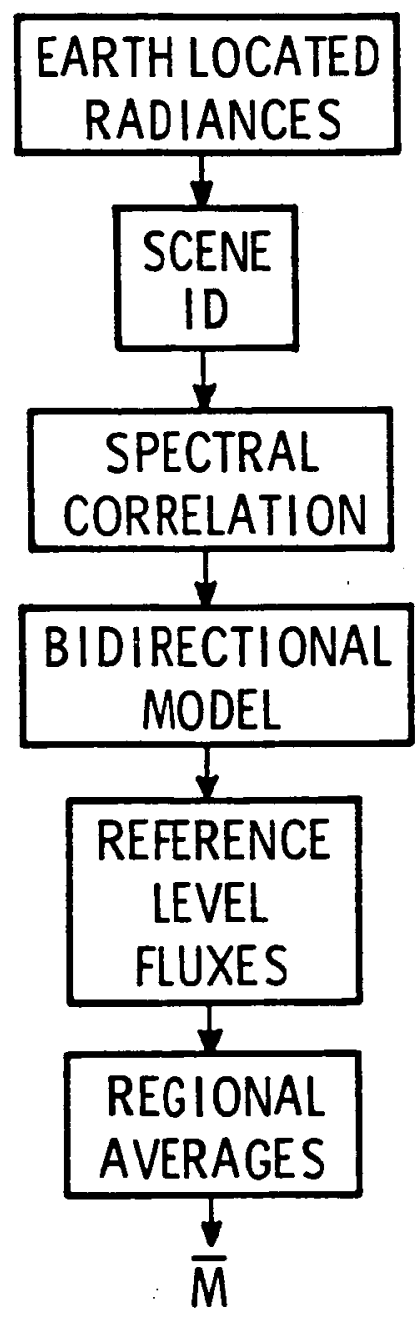

2. Inversion processing steps (Smith et al., 1986). 


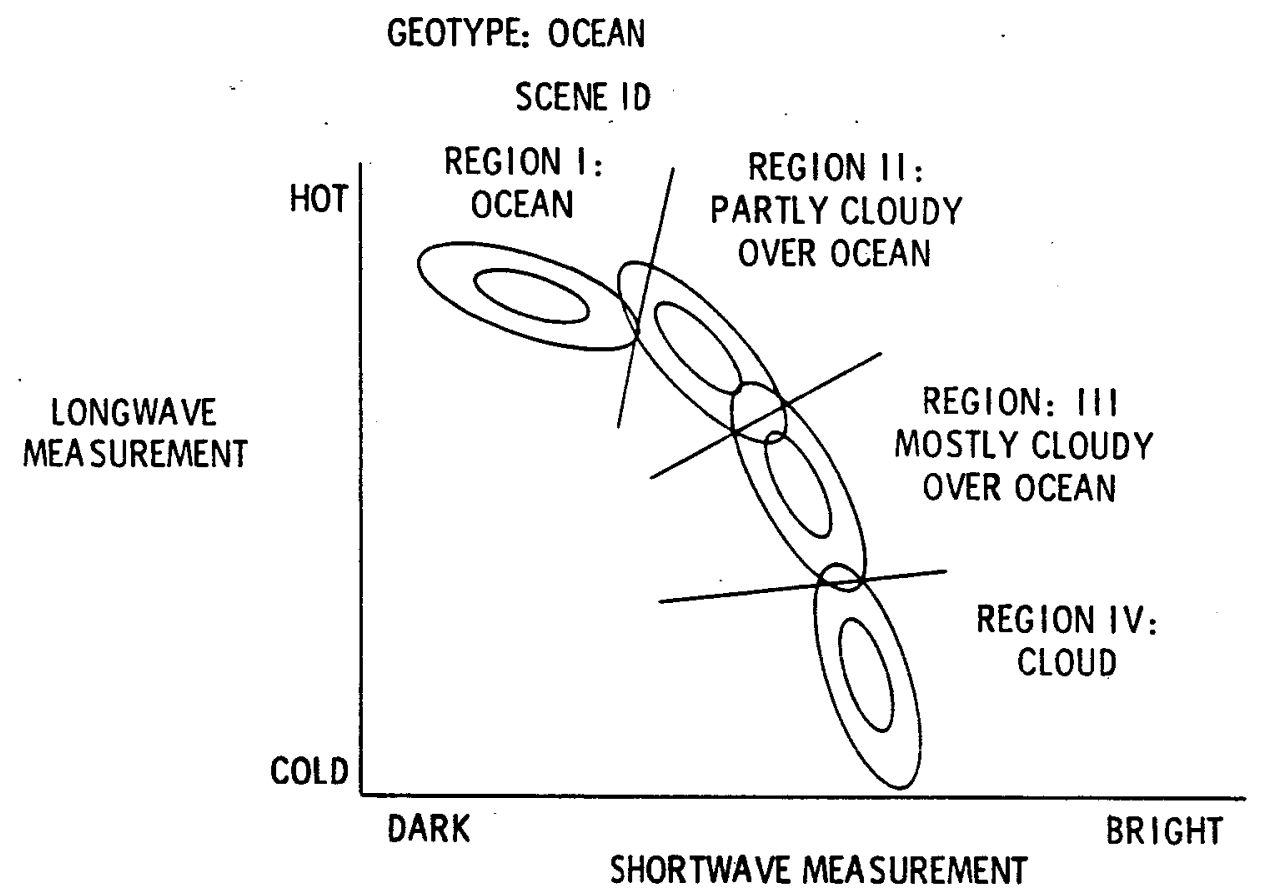

3. Schematic of maximum likelihood estimate (Smith et a1., 1986). 
Radiative transfer calculations

- $(5$ temperature profiles) -

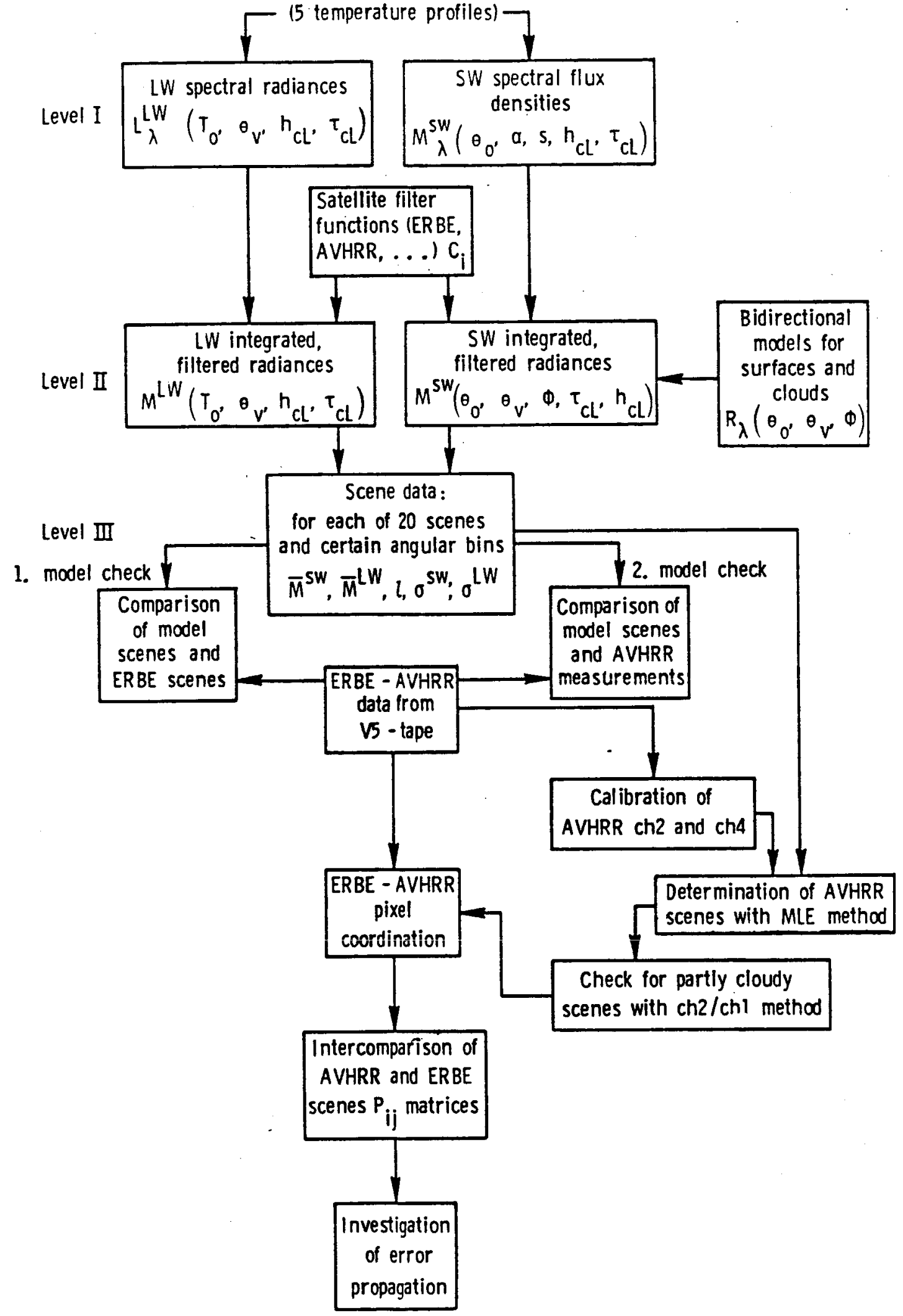

4. Schematic flow of simulation approach. 

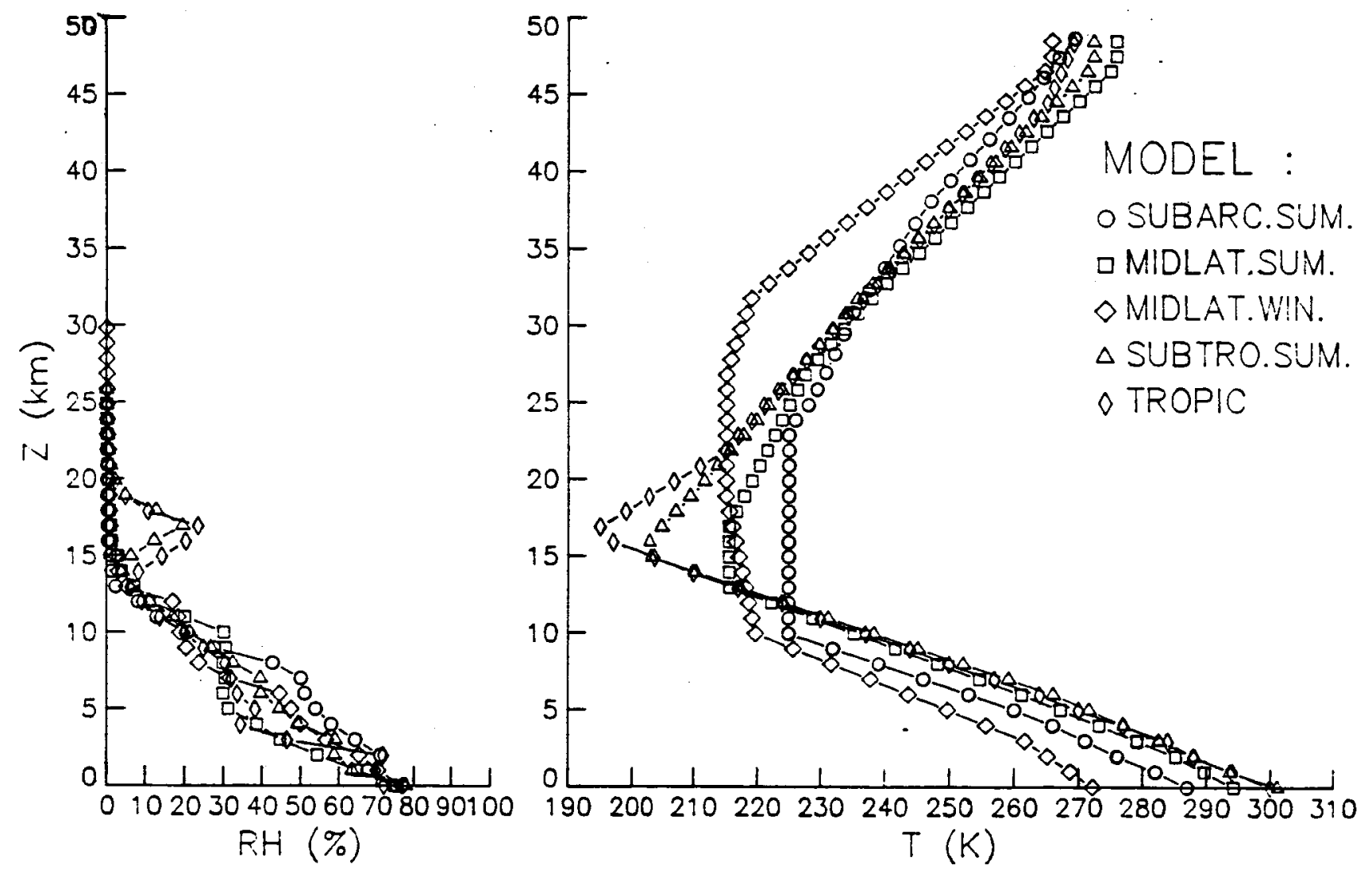

5. Vertical profiles of temperature and relative humidity for five atmospheric models (McClatchey, 1983) used in this study. 


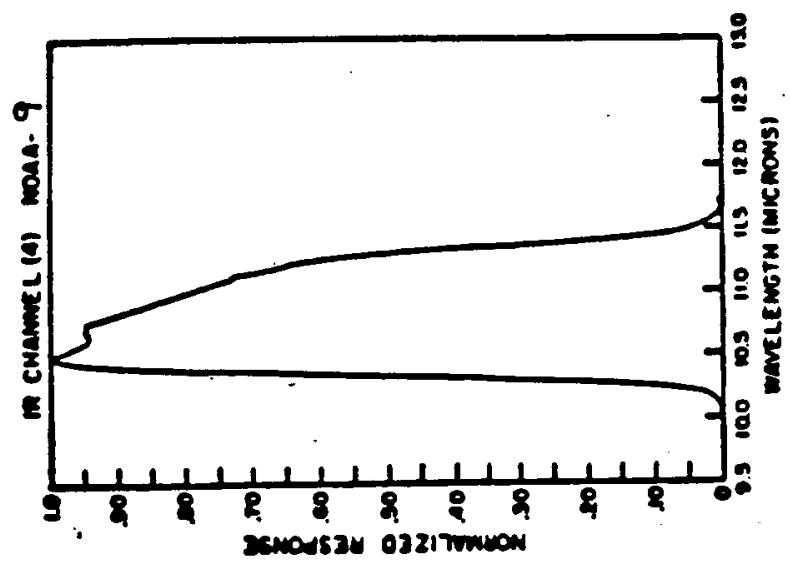

$\frac{5}{4}$

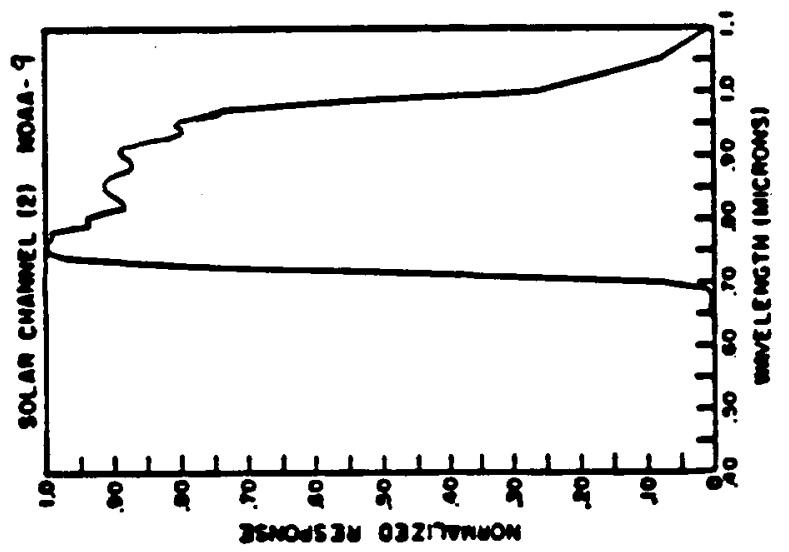

$\sim$

$a$

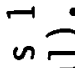

올 올

둥

它

몬

呈 年

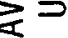

or

$5=$

$\sum \underset{0}{2}$

4 i

๑

มี

등으

응

ํํㄴ

농

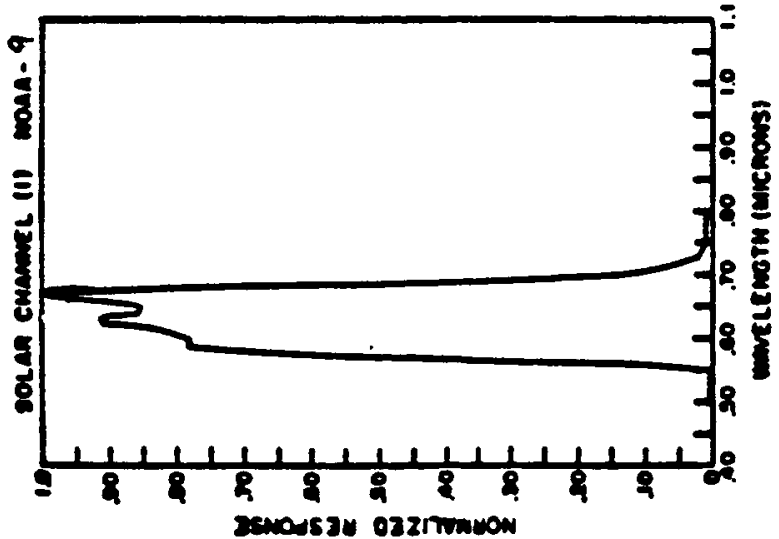

- 5

$\leqslant \leq$

U⿺

号

$\dot{0}$ 

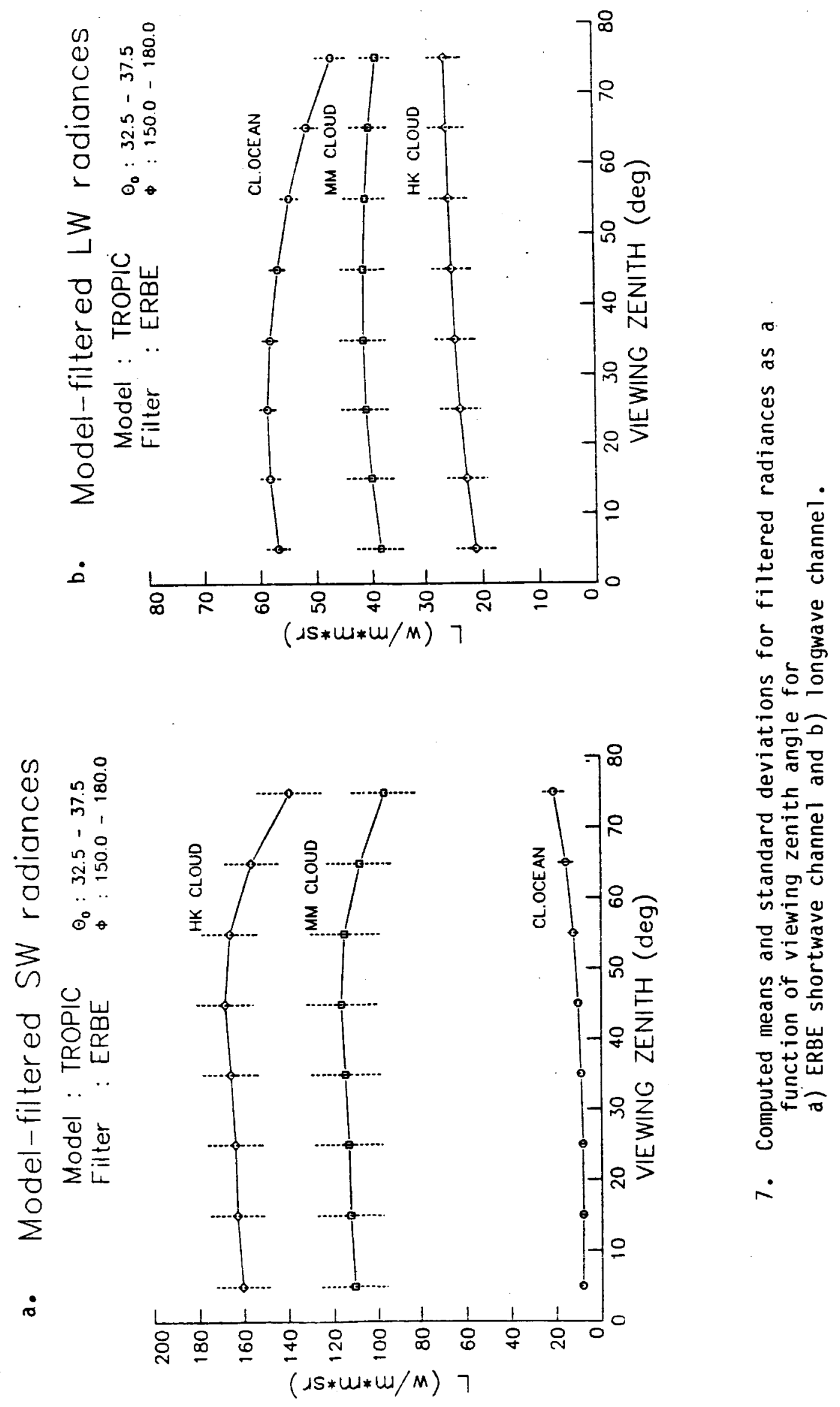


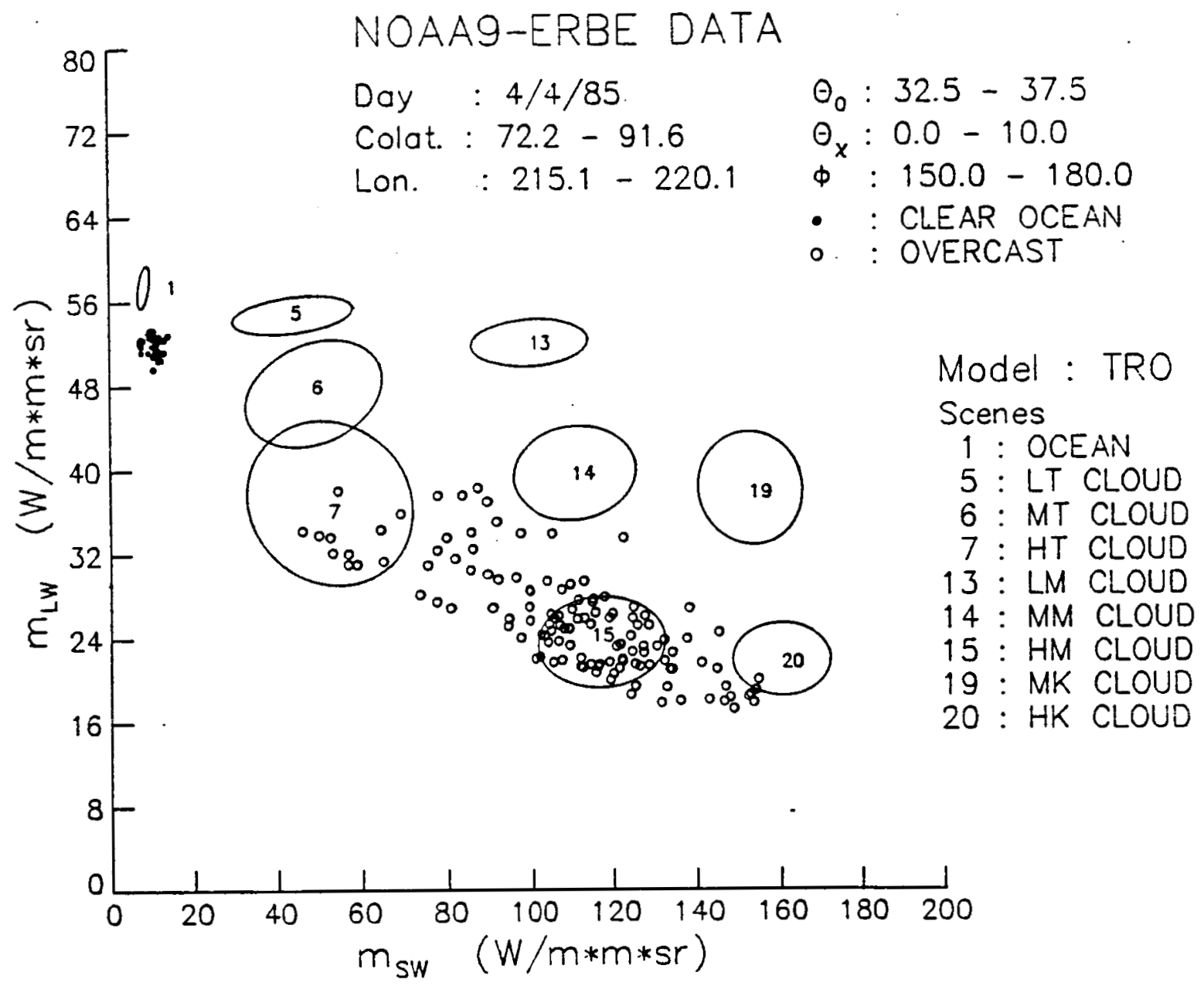

8. Bispectral distribution of ERBE measurements aboard NOAA 9 spacecraft and modeled one-sigma ellipses within for a sample case.

For April 4, 1985, area 1197 (see text). 


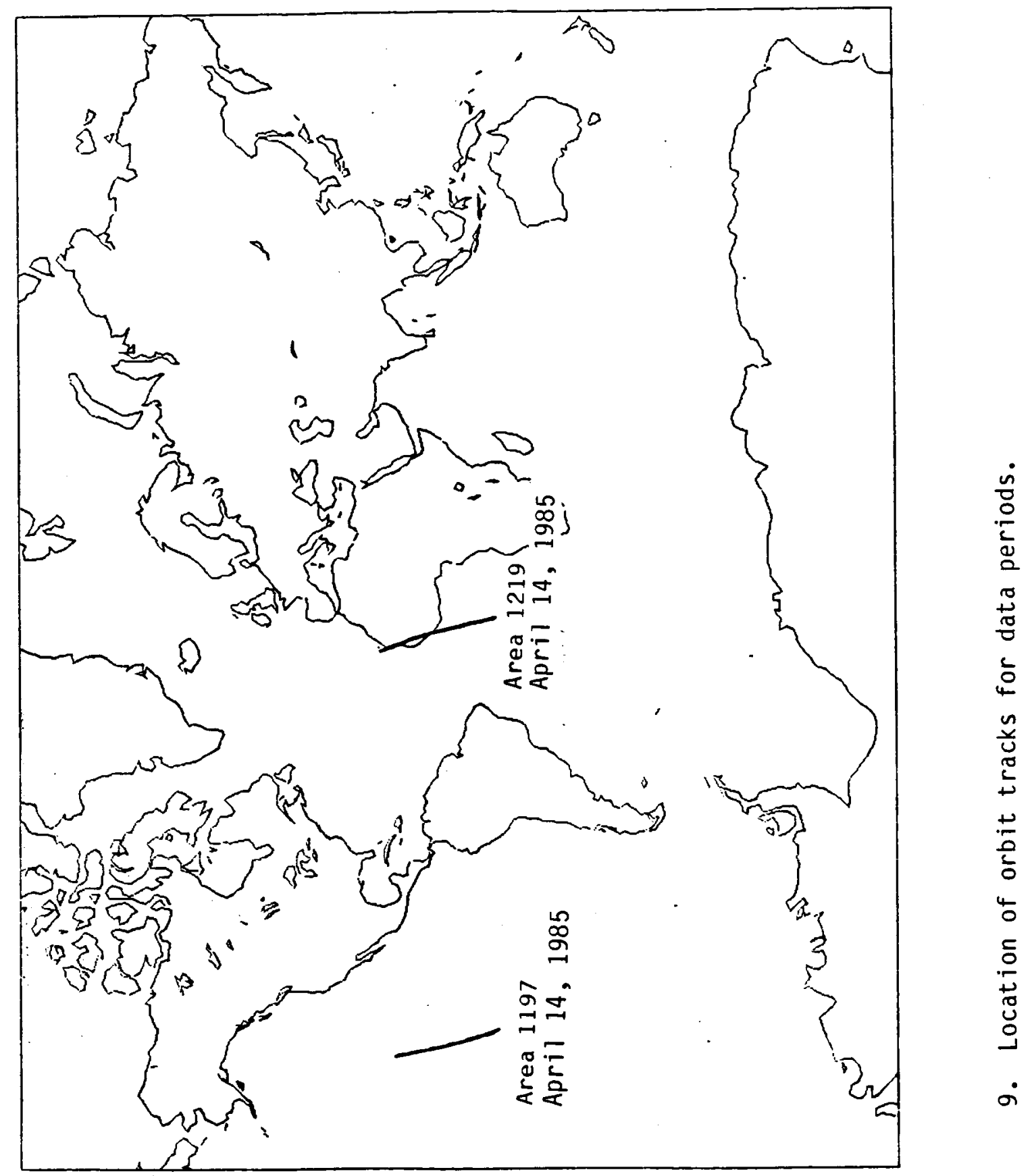


a.

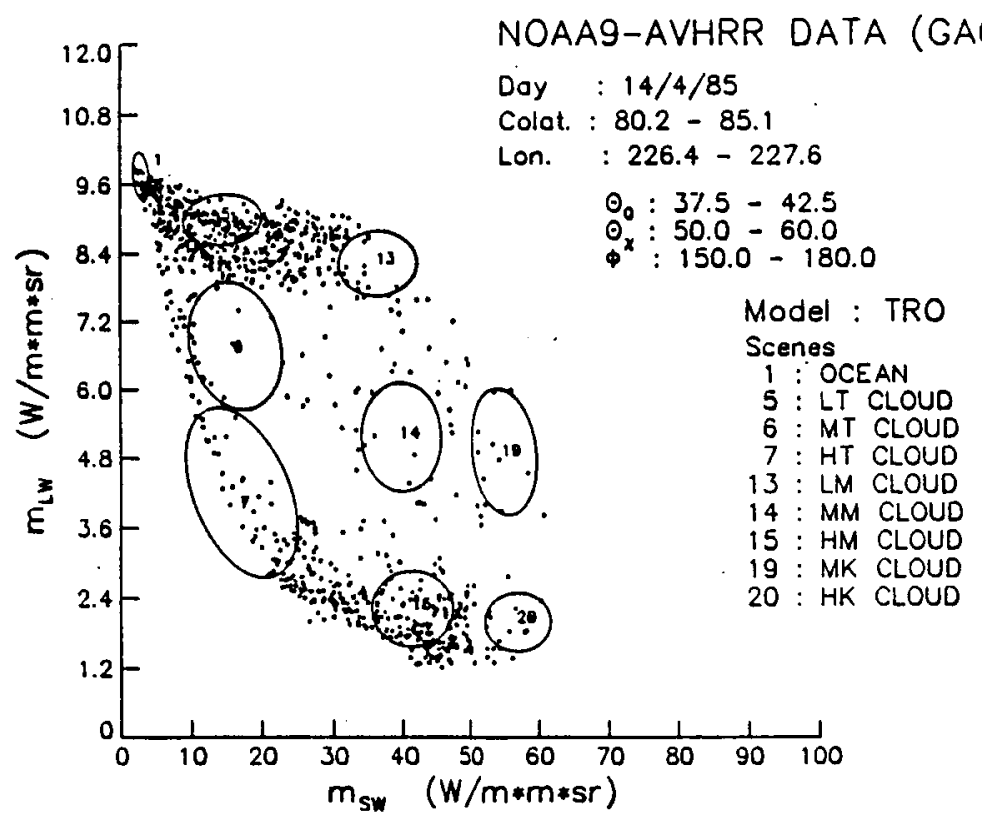

b.

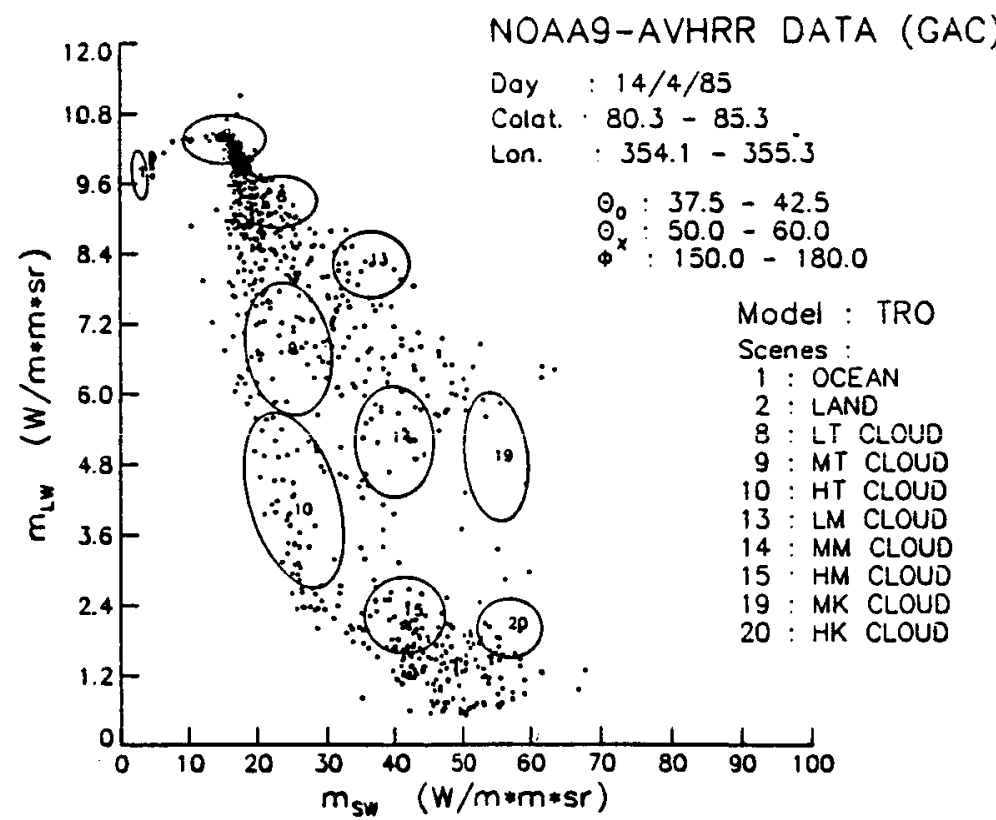

10. Bispectral distribution of AVHRR measurements aboard NOAA 9 spacecraft and modeled one-sigma ellipses within for a sample case.

a) for April 4, 1985, area 1197

b) for April 14, 1985, area 1219 (see text). 


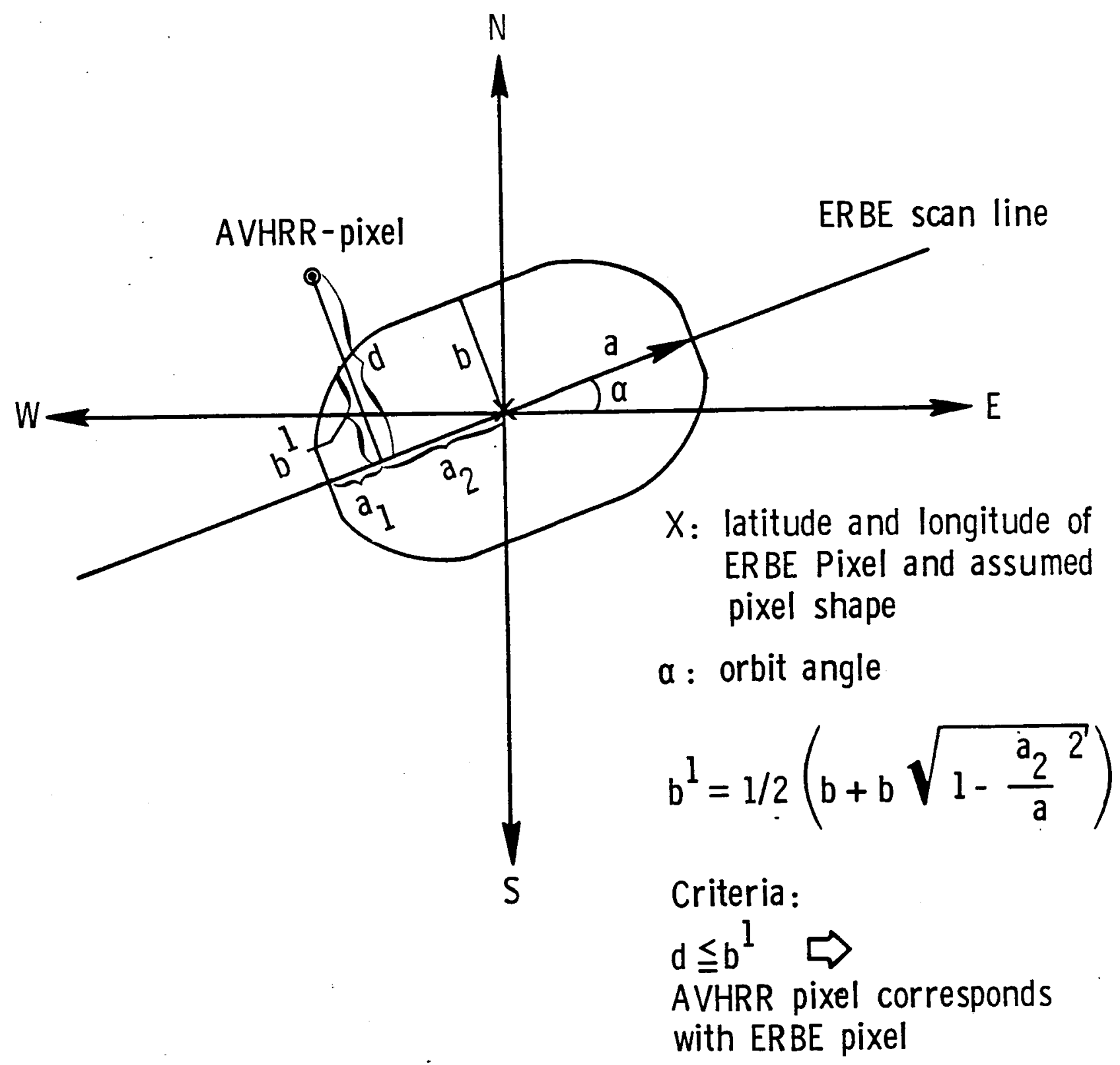

11. Geometry of mapping AVHRR pixels into ERBE pixels. 
AVHRR VIS-CHANNELS RATIO

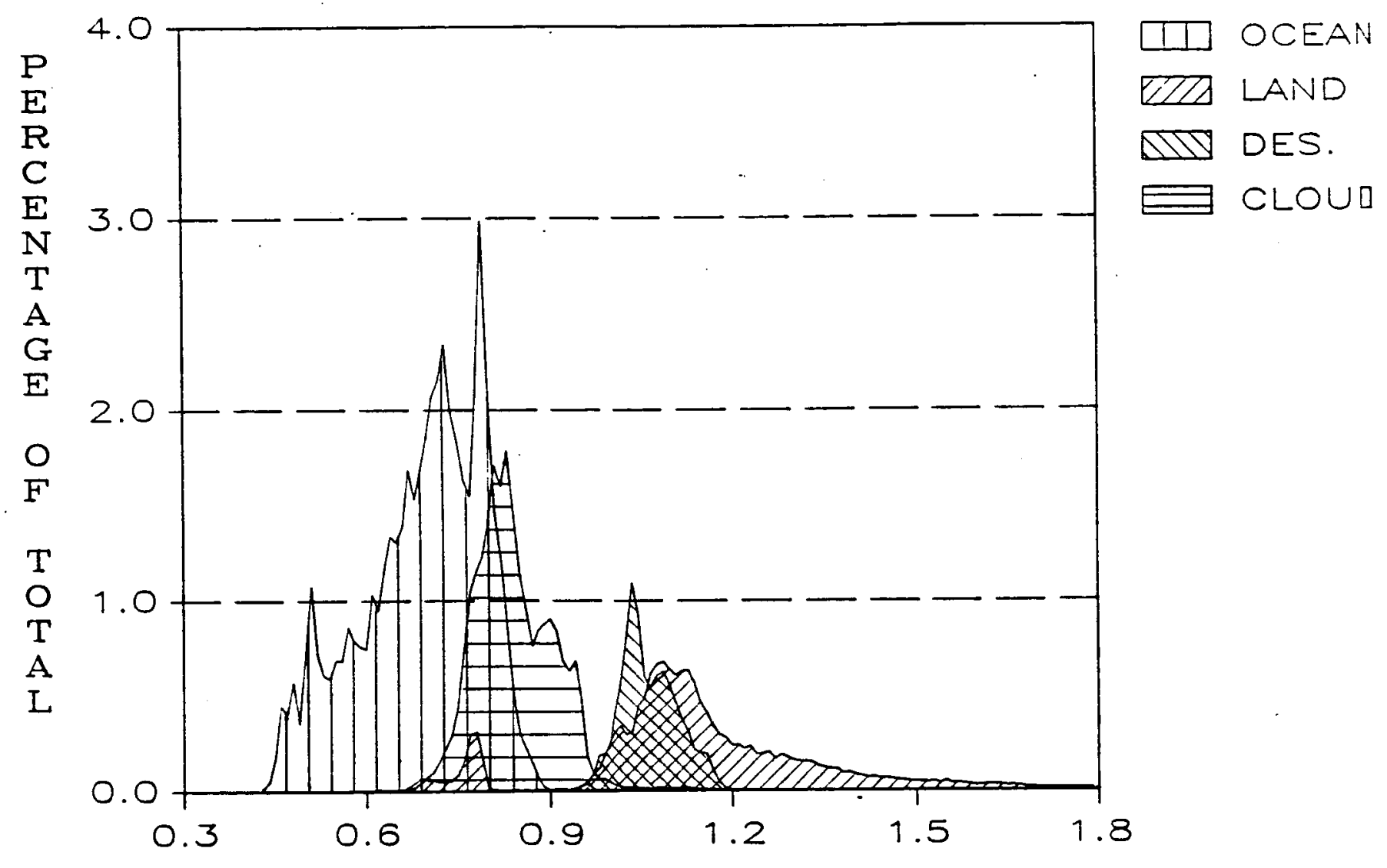

CHANNELZ/CHANNEL1

12. Histogram of ratio Channel $2 /$ Channel 1 for AVHRR data for differing scene types. 


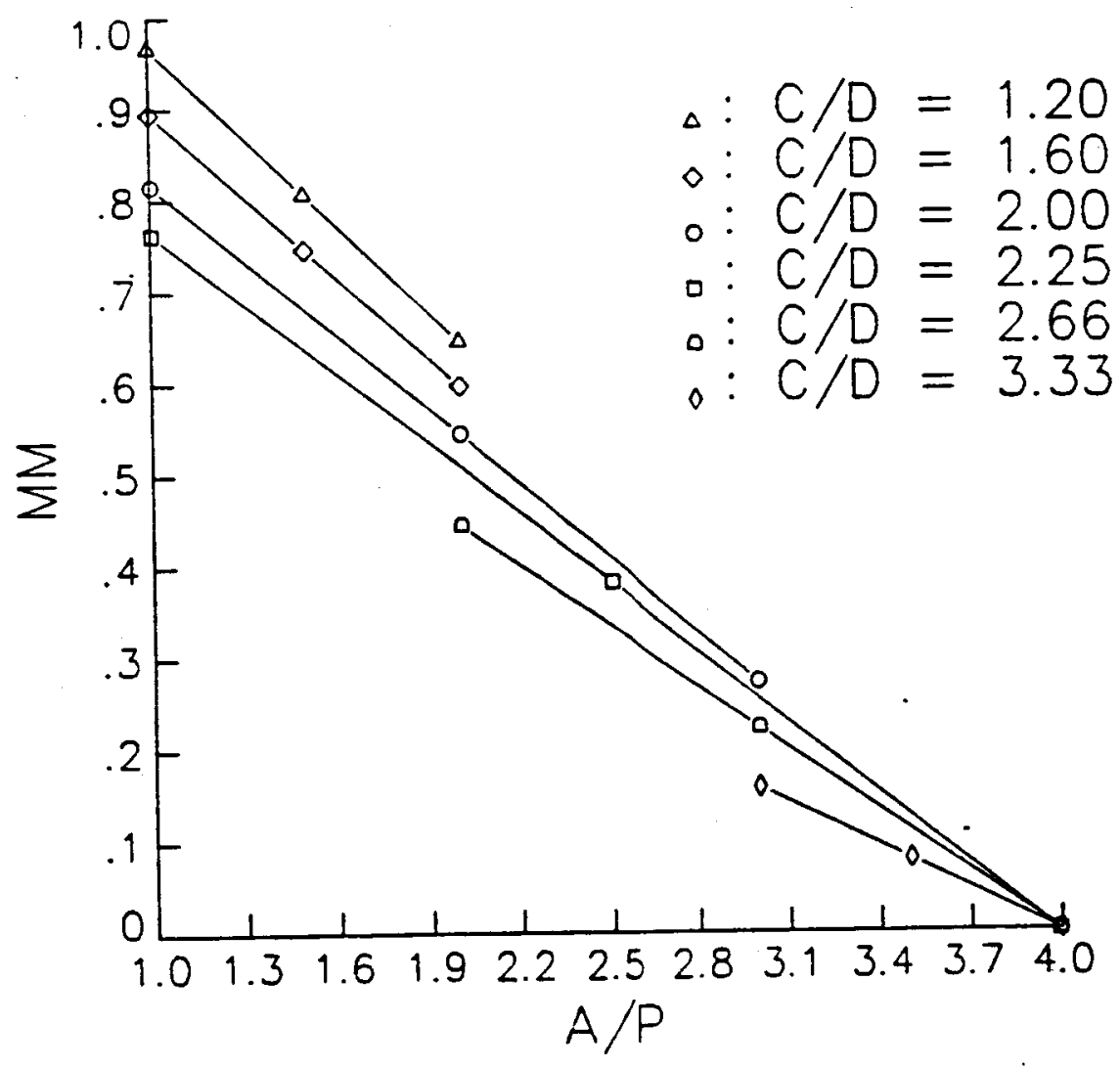

13. Dependence of matrix moment $M M$ on $A / P$ and $C / D$. 

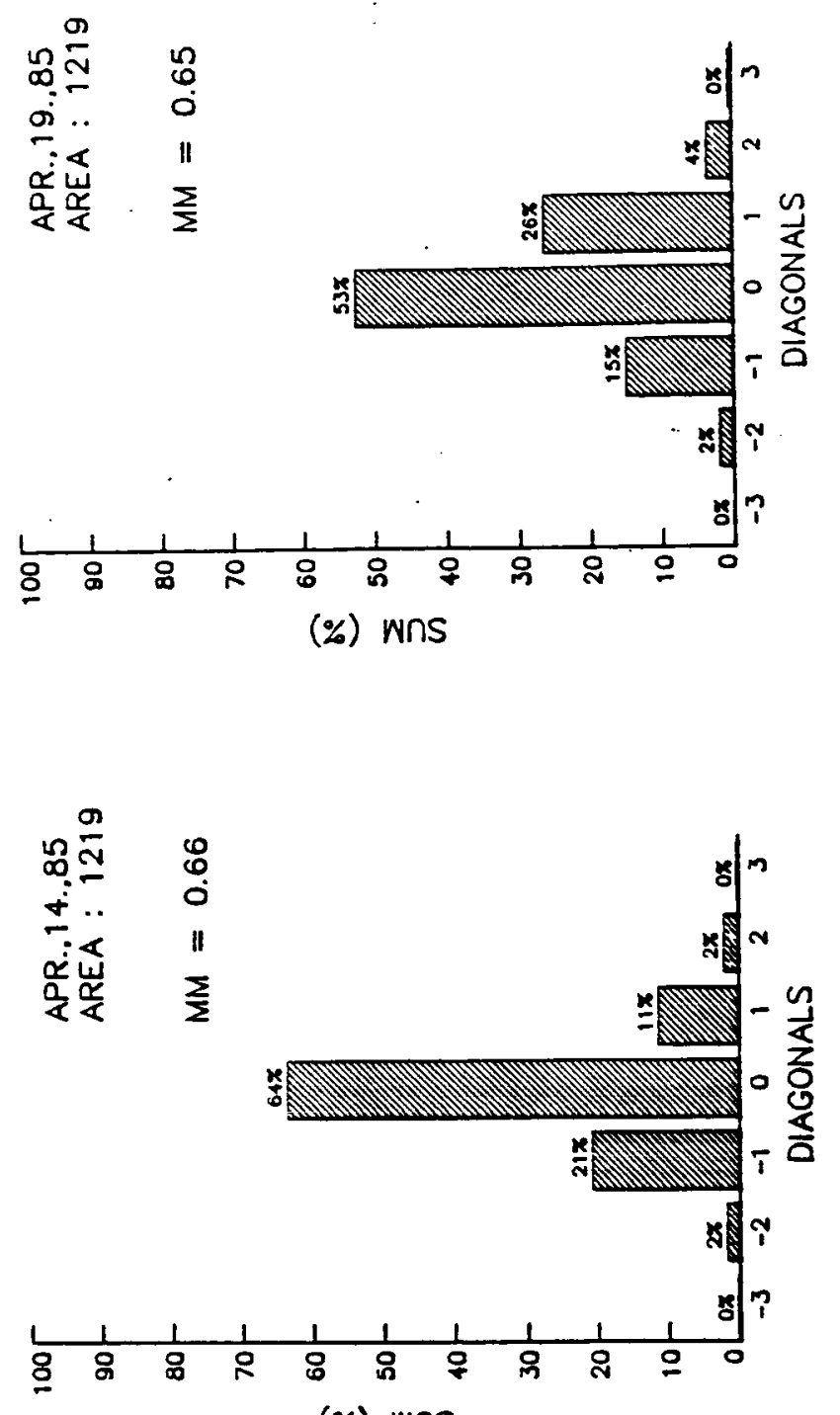

(\%) wns

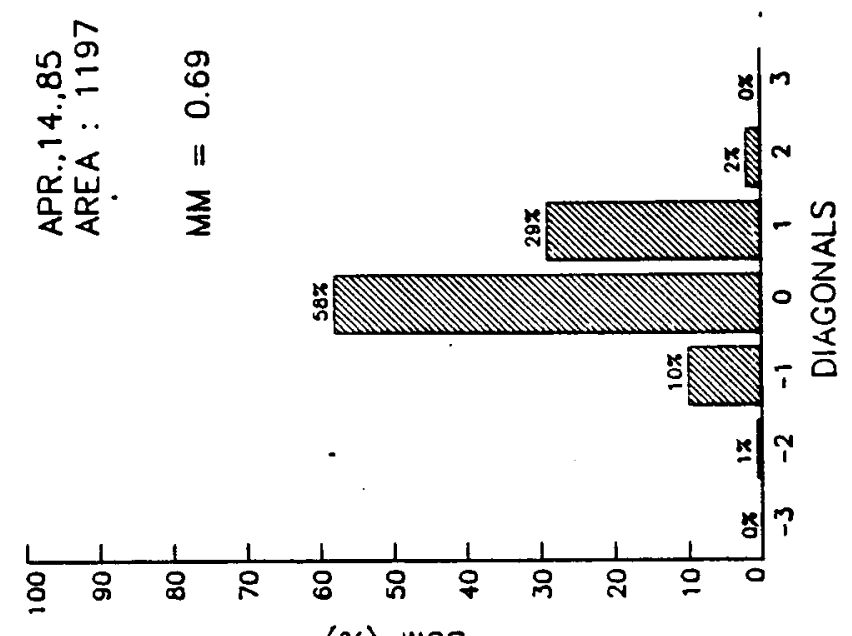

(\%) wnS

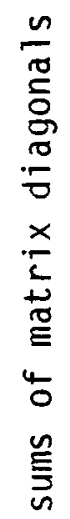

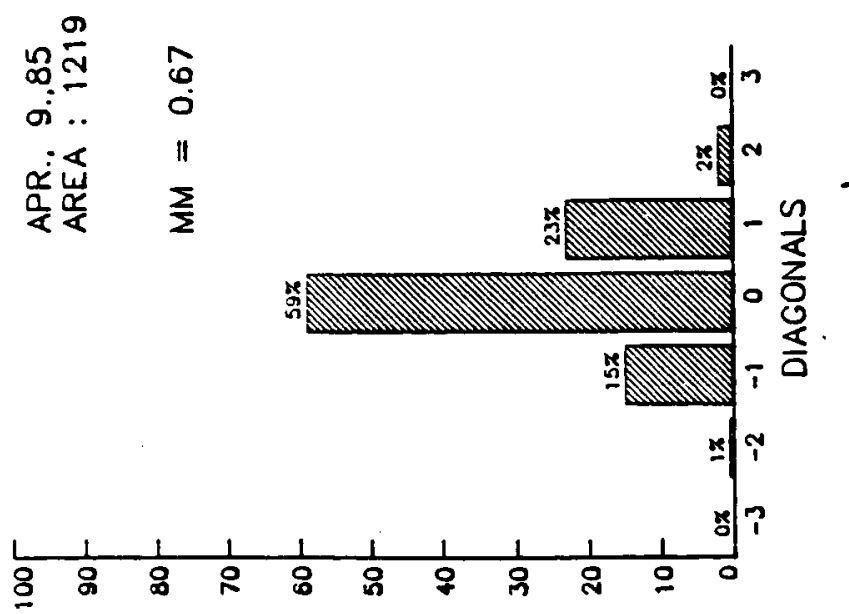

(๕) wnS

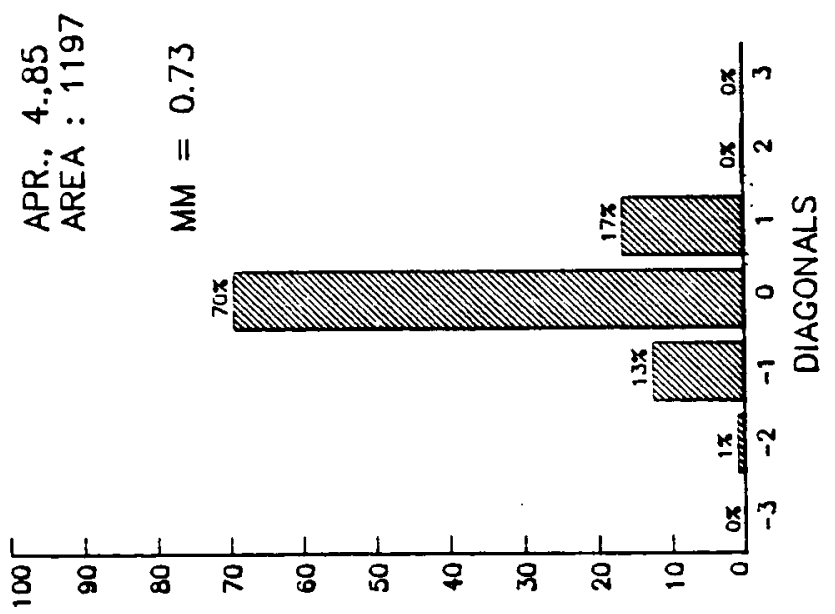

(\%) wกS 


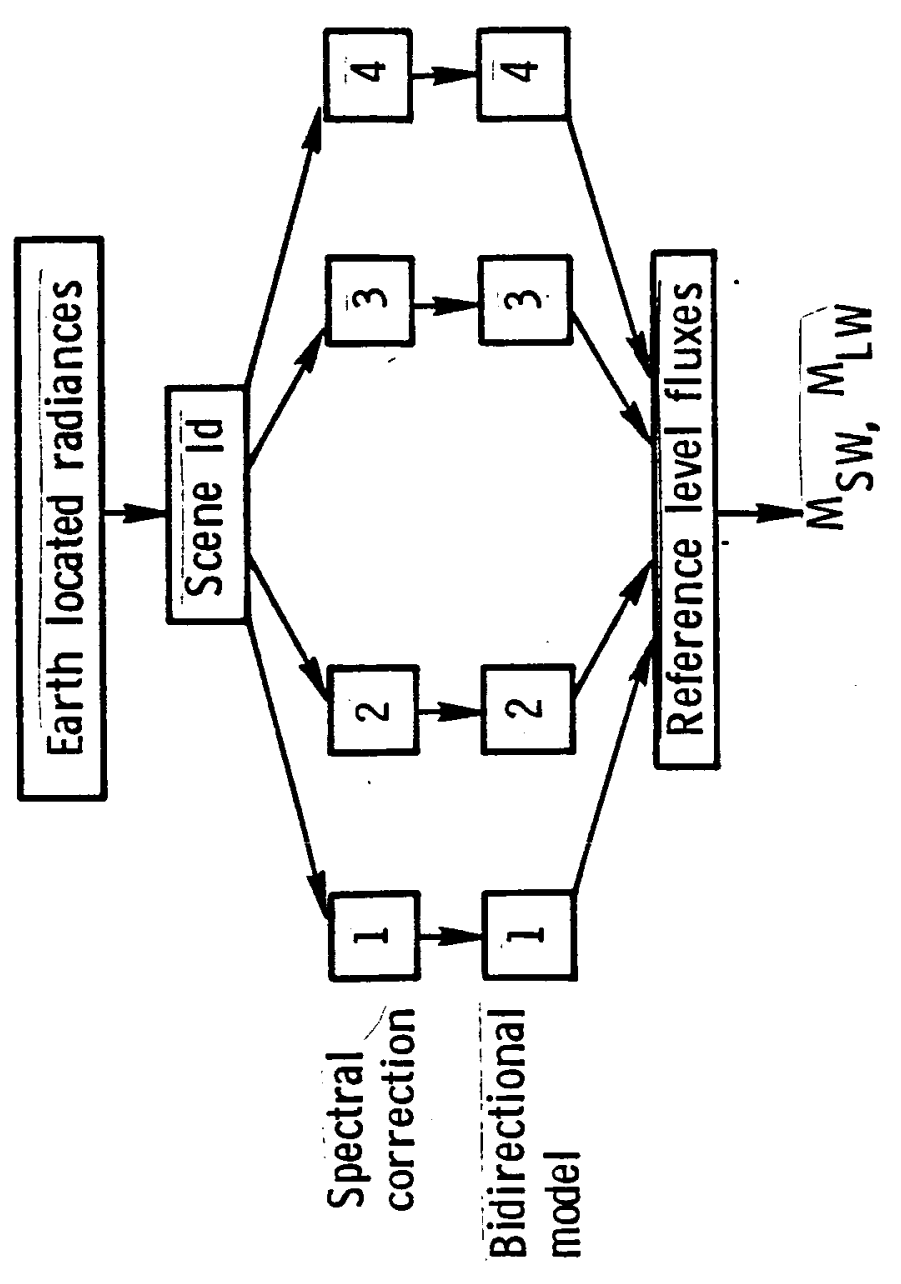

5
0
$\frac{1}{0}$
0
$-\frac{1}{4}$
$\frac{1}{5}$
$\frac{0}{0}$

巳ัย

$\stackrel{\circ}{+}$

$\stackrel{\Phi}{ }$

음

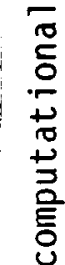
4

$\frac{0}{\frac{0}{E}}$

i 


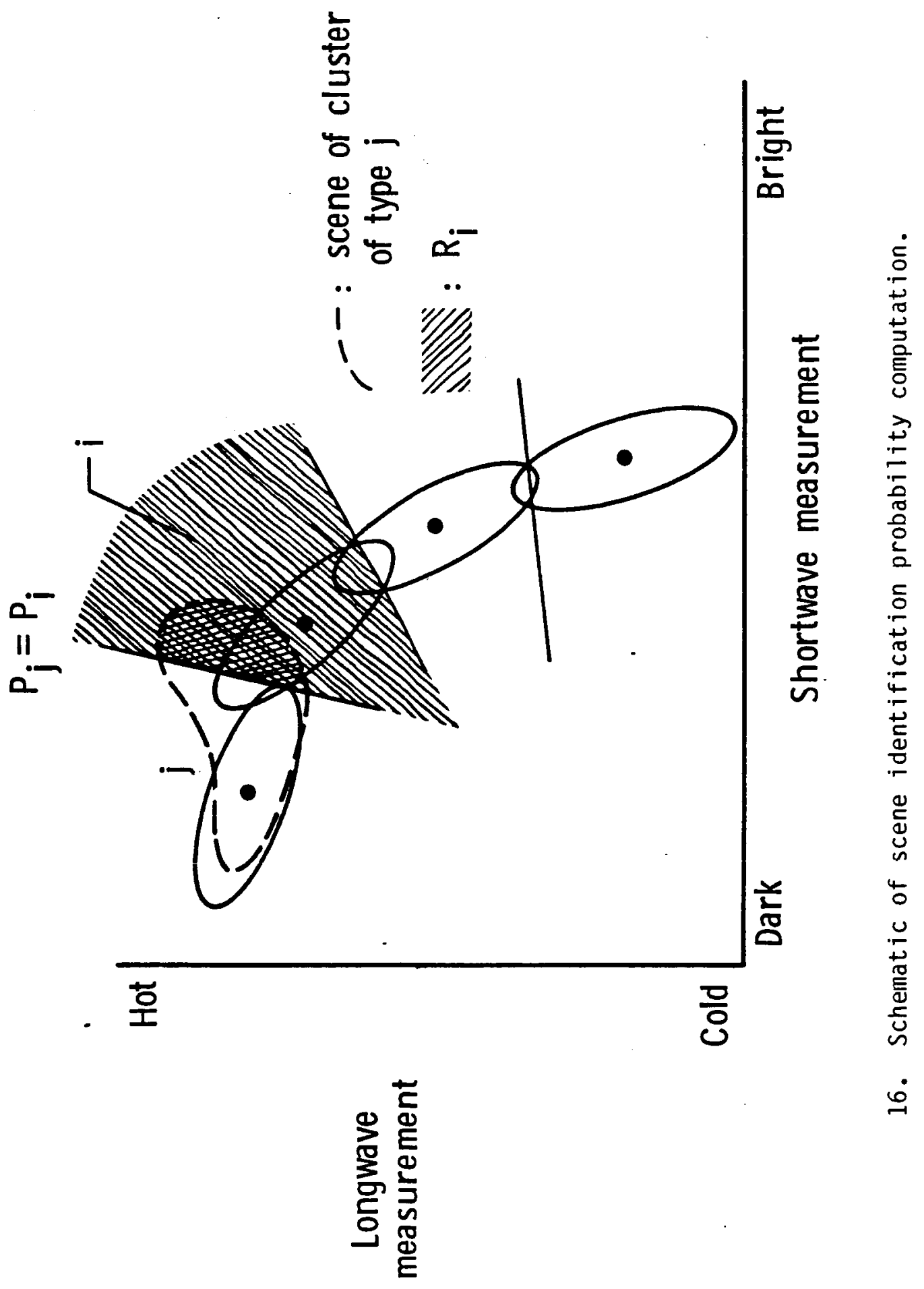




\section{SW exitance errors}

ERBE scenes

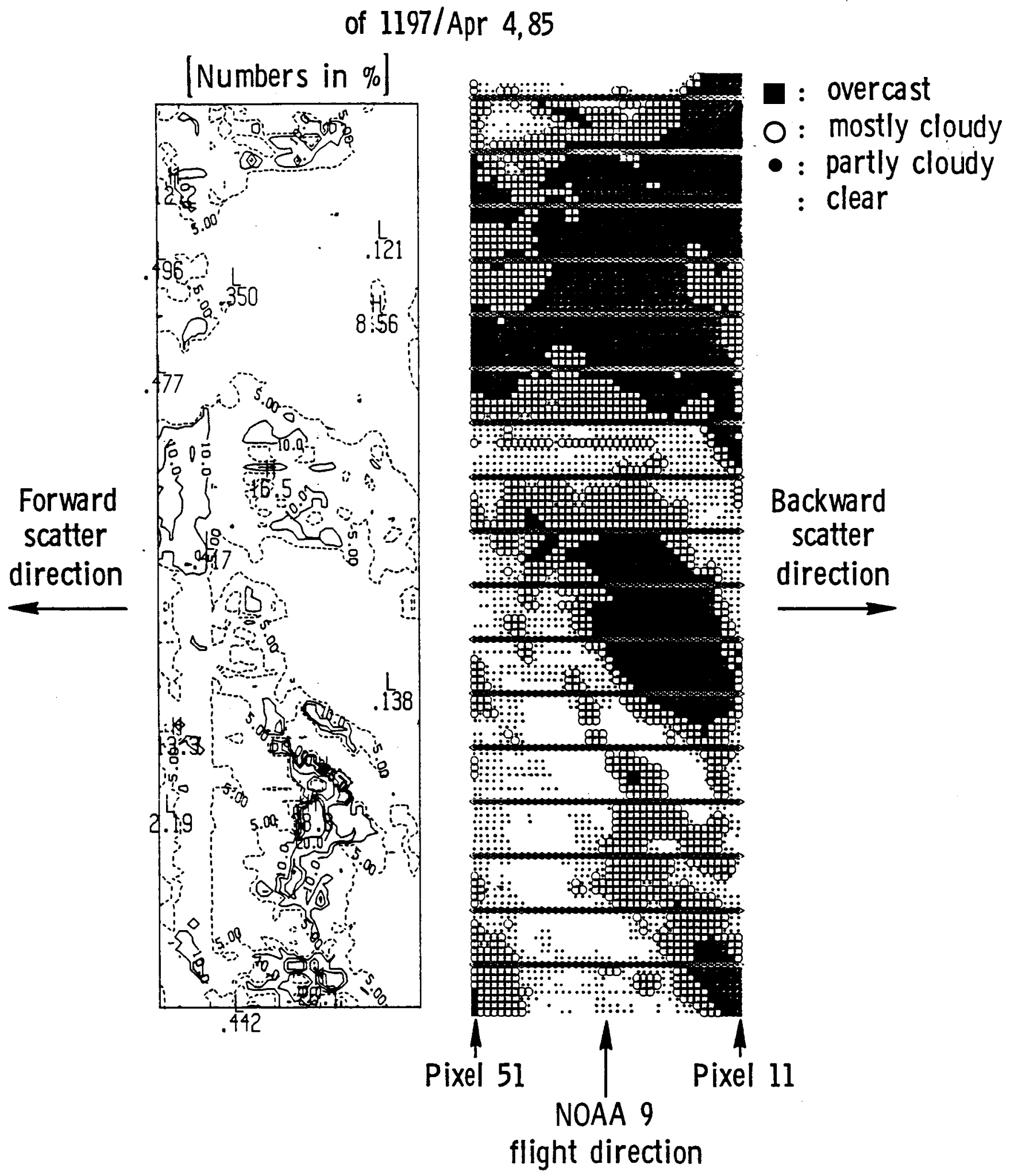

17. Standard deviation of shortwave radiant exitance errors due to scene identification errors. 